\title{
Differential Calculus for Differential Equations, Special Functions, Laplace Transform
}

\author{
Do Tan $\mathrm{Si}^{1}$ \\ ${ }^{1}$ HoChiMinhcity Physical Association, Vietnam, 40 Dong Khoi, Q1, HochiMinhcity, Vietnam \\ Correspondence: Do Tan Si, HoChiMinhcity Physical Association, Vietnam, 40 Dong Khoi, Q1, HochiMinhcity, \\ Vietnam. E-mail: tansi_do@yahoo.com
}

Received: January 20, 2015 A ccepted: January 27, 2016 Online Published: January 29,

2016 doi:10.5539/apr.v8n1p158 URL: http://dx.doi.org/10.5539/apr.v8n1p158

\section{Abstract}

A new definition of the inverse operator of any operator which applies a space of differentiable functions onto itself is proposed and a formula changing the operator $f(A) g(B)$ where $A B-B A \equiv \hat{I}$ into a sum of operators $\frac{1}{k !} g^{(k)}(B) f^{(k)}(A)$ is proved. Thank to this relation between operators some formulae in integral calculus are found; a new and rapid method for resolutions of differential equations teached in universities is exposed in details. It is seen to be useful also for obtaining the differential operators $\operatorname{say} \exp \left(-\frac{1}{4} D_{x}^{2}\right),\left(D_{x}-1\right)^{n},(-)^{n}\left(1-D_{x}\right)^{n+\alpha}$, $(2 \lambda)_{n}{ }_{0} F_{1}\left(-; \frac{1}{\lambda+1 / 2} ;-\frac{B^{2}}{4}\right), \cos B$ that transform monomials into Hermite, Laguerre, associated Laguerre, Gegenbauer, Chebyshev polynomials and for getting quasi all their main properties in a very concise manner. Is proposed also the differential representation of the Laplace transform permitting the differential calculus to prove consicely its properties.

Keywords: Operational calculus, Inverse operators, Differential equations, Special functions, Laplace transform, Eigenfunctions, Newton binomial.

\section{Introduction}

An application of a space of functions into itself is represented by an operator. In this work we will consider operators representing linear applications of the space $\mathcal{D}$ of differentiable functions onto itself

$$
\begin{aligned}
A(f(x)+g(x))= & A f(x)+A g(x) \quad f, g, A f, A g \in \mathcal{D} \\
& A(c f(x))=c A f(x)
\end{aligned}
$$

The well known operators are certainly the derivative operator $D_{x}$, the Eckart "multiply by the argument x" operator $\hat{X}$ (Eckart, 1926) and the identity operator $\hat{I}$

$$
\begin{array}{ll}
D_{x} f(x)=f^{\prime}(x) & \forall f(x) \\
\hat{X} f(x)=x f(x) & \forall f(x) \\
\hat{I} f(x)=f(x) & \forall f(x)
\end{array}
$$

The first remark to be notified is that $A$ acts on the whole product of functions at his right

$$
A f(x) g(x)=A f((x) g(x))
$$

The second remark is that $A 0$ may be different from 0 for example

$$
e^{x} \int e^{-x} 0=c e^{x}
$$


so that in general $A f$ is defined only within $A 0$

$$
A f(x)=A(f(x)+0)=A f(x)+A 0
$$

Profoundly we think that (1.6) means that there is not at all determinism in a universe where the number zero exists.

Two operators $A$ and $B$ are said equivalent if they give the same result when acting on an arbitrary function

$$
A \equiv B \Leftrightarrow A f(x)=B f(x) \quad \forall f
$$

From this definition one may define the addition and the multiplication of operators as following

$$
\begin{gathered}
C \equiv A+B \Leftrightarrow C f(x)=A f(x)+B f(x) \quad \forall f \\
C \equiv A B \Leftrightarrow C f(x)=(A B) f(x)=A(B f(x))=A B f(x) \quad \forall f
\end{gathered}
$$

Afterward one may define the operators $A^{n}, A^{n / m}$ where n, m are positive integers and series of them. An example is the definition of $D_{x}^{v}$ by Riemann (Oldham \& Spanier, 1974)

$$
D_{x}{ }^{\mu} x^{v}=\frac{\Gamma(v+1)}{\Gamma(v-\mu+1)} x^{\nu-\mu} ; \quad v, \mu \in \mathrm{C}
$$

Two operators in general may do not commute i.e. $A B$ may be different from $B A$.

In order to clarify the above assertion, let us examine the following analysis.

Because

$$
D_{x} \hat{X} f(x)=D_{x} x f(x)=x f^{\prime}(x)+f(x)=\left(\hat{X} D_{x}+\hat{I}\right) f(x) \quad \forall f
$$

we get the relation between two operators

$$
D_{x} \hat{X} \equiv \hat{X} D_{x}+\hat{I}
$$

which shows that $D_{x}$ and $\hat{X}$ do not commute.

From now on a relation between operators will be called identity and that between two functions formula.

There exists the notion of commutator of an ordered couple of operators $(A, B)$ defined as follows

$$
[A, B] \equiv A B-B A
$$

For example

$$
\left[D_{x}, \hat{X}\right] \equiv D_{x} \hat{X}-\hat{X} D_{x} \equiv \hat{I}
$$

Differential calculus consists in utilizing operators constructed from $D_{x}, \hat{X}, \hat{I}$ to study differential and partial differential equations, special functions and so on as we can find abundantly in literature from the time Heaviside (1893) published his invention of operational calculus. The non exhaustive list of references is (Eckart, 1926; Wilcox, 1967; Abramovitz \& Stegun, 196; Wolf, 1976; Do, 1978) and many others may be founded on the net by searching for operational calculus, hyperdifferential operators, etc.....

In a recent work we have studied the transforms of functions and operators by exponential in linear and quadratic operators in $D_{x}$ and $\hat{X}$, named hyperdifferential operators (Do, 2015).

Considering utilizing ordinary operators in differential calculus we meet firstly a huge difficulty which consists in the impossibility to define the inverse $A^{-1}$ of an operator $A$ by the identity

$$
A^{-1} A \equiv A A^{-1}
$$

in the case where $A$ has the properties

$$
A 0=0
$$

and there exists a function $V(x) \neq 0$ such that

$$
A V(x)=0
$$

because in this case we have simultaneously 


$$
A^{-1} 0=A^{-1} A V(x)=V(x)
$$

and

$$
A^{-1} 0=A^{-1} A 0=0
$$

which is paradoxal.

And, without $A^{-1}$ one can't resolve the differential equation $A y=f(x)$ by writing

$$
y=A^{-1} f(x)
$$

The second difficulty is the lack of an identity which would help to put any operator $f\left(D_{x}\right) g(\hat{X})$ into a sum of operators having the form $u(\hat{X}) v\left(D_{x}\right)$ in order to deplace some operator inside a product of non commutative operators; to factorize a sum of operators such as $\left(D_{x}+f(\hat{X})\right)$ then invert it; to simplify the calculations of $f\left(D_{x}\right) g(x) h(x)=f\left(D_{x}\right) g(\hat{X}) h(x)$, etc...

Willing to bypass these difficulties, this work aims to propose a free of paradox definition of the inverse operator $A^{-1}$ of any operator $A$ and to establish the fundamental identity of operational calculus

$$
f(D) g(X)=\sum_{k=0}^{\infty} \frac{1}{k !} g^{(k)}(X) f^{(k)}(D)
$$

where $(D, X)$ is a couple operator obeying the condition $(D X-X D) \equiv \hat{I}$.

Afterward in order to convince readers and students about the worth of differential calculus we will apply these propositions to obtain formulae in the field of differential and integral calculus, to study the Laplace transform, to simplify greatly the resolution of differential equations teached in college and to get the differential operators which transform monomials into the Hermite, Laguerre, Gegenbauer, Chebyshev polynomials. Thank to these representative operators the main properties of these polynomials are deduced in a very concise manner.

\section{The Inverse Operator of an Opertor}

\subsection{Definition}

Given $A$ and let $V_{i}(x)$ be one of the solutions of the differential equation

$$
A y=0
$$

Firstly we denote by $A^{-1} 0$ the general solution of the above equation

$$
A^{-1} 0=\sum_{i} c_{i} V_{i}(x) \quad \forall c_{i}
$$

The entity $A^{-1} 0$ is seen to be the kernel of $A$

$$
A A^{-1} 0=\sum_{i} c_{i} A V_{i}(x)=0 \quad \forall c_{i}
$$

and has the particular property

$$
A^{-1} 0=A^{-1}(c 0)=c A^{-1} 0 \quad \forall c \neq 0
$$

Secondly we propose to define the inverse operator $A^{-1}$ of an operaror $A$ by the relation

$$
A^{-1} A f(x)=f(x)+A^{-1} 0 \quad \forall f
$$

With respect to this definition the primitive operator $\int$ defined by

$$
\begin{gathered}
\int 0=1 \\
\int f(x)=F(x) \Leftrightarrow D_{x} F(x)=f(x) \quad \forall f
\end{gathered}
$$

is the inverse of $D_{x}$ and vice versa 


$$
\begin{gathered}
\int D_{x} f(x)=f(x)+\int 0 \\
D_{x} \int f(x)=f(x)+D_{x} 0
\end{gathered}
$$

so that from now on we may write

$$
D_{x}^{-1} \equiv \int
$$

For completion we remark that if we may write

$$
D_{x}^{-1} 0=\int 0=c
$$

this is because $0=c 0$ and not because $D_{x}^{-1}$ is undetermined.

\subsection{Properties}

(i) From the definition of inverse operators (2.5) we have

$$
\begin{aligned}
& A^{-1} A 0=A^{-1} 0 \\
& A^{-1} A A^{-1} A 0=A^{-1} A 0+A^{-1} 0=A^{-1} A A^{-1} 0=2 A^{-1} 0
\end{aligned}
$$

and may conclude that

$$
\begin{gathered}
A 0=0 \Rightarrow A^{-1} 0=A^{-1} 0 \Rightarrow A^{-1} 0 \text { may be } \neq 0 \\
A^{-1} 0 \neq 0 \Rightarrow A^{-1} A 0=A^{-1} 0 \Rightarrow A 0=0 \\
A 0 \neq 0 \Rightarrow A^{-1} 0=0 \text { unless paradoxal with the previous assertion. }
\end{gathered}
$$

(ii) If $A^{-1}$ is the inverse of $A$ then $A$ is the inverse of $A^{-1}$

$$
\left(A^{-1}\right)^{-1} \equiv A
$$

Indeed, appplying $A$ on both members of (2.15) we see that

$$
A A^{-1}(A f(x))=A\left(f(x)+A^{-1} 0\right)=A f(x)+A A^{-1} 0
$$

For $A 0=0$ we get

$$
A A^{-1}(A f(x))=A f(x)+0=A f(x)+A 0 \quad \forall f
$$

For $A 0 \neq 0$ we have $A^{-1} 0=0$ and also get

$$
A A^{-1}(A f(x))=A\left(f(x)+A^{-1} 0\right)=A f(x)+A 0
$$

(iii) $A^{-n}$ is the inverse of $A^{n}$

Let

$$
A^{-n} \equiv A^{-1} A^{-1} \ldots A^{-1} \quad n \text { times }
$$

we will prove by recursion that $A^{-n}$ is the inverse of $A^{n}$.

For this purpose suppose that

$$
A^{-n} A^{n} f(x)=f(x)+A^{-n} 0
$$

so that 


$$
\begin{aligned}
A^{-n-1} A^{n+1} f(x) & =A^{-n} A^{-1} A A^{n} f(x)=A^{-n}\left(A^{n} f(x)+A^{-1} 0\right) \\
& =f(x)+A^{-n} 0+A^{-n-1} 0
\end{aligned}
$$

Remarking that

$$
A^{-n-1} 0=A^{-n}\left(A^{-1} 0+0\right)=A^{-n-1} 0+A^{-n} 0
$$

we finally get

$$
A^{-n-1} A^{n+1} f(x)=f(x)+A^{-n-1} 0 \quad \text { QED }
$$

By the way we notify that

$$
\begin{gathered}
A^{-n-1} 0=A^{-n-1} 0+A^{-n} 0=A^{-n-1} 0+A^{-n} 0+\ldots+A^{-1} 0 \\
\text { (iv) } A^{n+m} \equiv A^{n} A^{m} \quad \forall n \geq 0, m \geq 0
\end{gathered}
$$

This is because both members are $(\mathrm{n}+\mathrm{m})$ times products of $A$.

$$
A^{-n-m} \equiv A^{-n} A^{-m} \forall n \geq 0, m \geq 0
$$

This is because both members are $(\mathrm{n}+\mathrm{m})$ times products of $A^{-1}$

(v) For $A 0=0$ we have $A A^{-1} \equiv \hat{I}$

so that

$$
A^{n-m} \equiv A^{n} A^{-m} \quad \forall n \geq 0, m \geq 0
$$

Nevertheless if $A 0=0$ but $A^{-1} 0 \neq 0$

$$
A^{-n} A^{+m} \neq A^{-n+m}
$$

because in this case

$$
\begin{aligned}
A^{-n} A^{+m} f(x)=A^{-n} A^{+n} A^{-n+m} f(x) & =A^{-n+m} f(x)+A^{-n} 0 \quad \forall m \geq n \geq 0 \\
A^{-n} A^{+m} f(x)=A^{-n+m} A^{-m} A^{+m} f(x) & =A^{-n+m} f(x)+A^{-n+m} A^{-m} 0 \\
& =A^{-n+m} f(x)+A^{-n} 0 \quad \forall n \geq m \geq 0 .
\end{aligned}
$$

With respect to the above properties we get the global formula valuable when $A 0=0$

$$
A^{p} A^{q} f(x)=A^{p} A^{q}(f(x)+0)=A^{p+q} f(x)+A^{p} A^{q} 0 \quad \forall p, q \in Z
$$

2.3 Inversion of a Product of Operators $A B$

From the definition of inverse operators (2.5) we may write

$$
\left(B^{-1} A^{-1}\right) A B f(x)=B^{-1}\left(B f(x)+A^{-1} 0\right)=f(x)+B^{-1} 0+B^{-1} A^{-1} 0
$$

Because

$$
B^{-1} A^{-1} 0=B^{-1}\left(A^{-1} 0+0\right)=B^{-1} A^{-1} 0+B^{-1} 0
$$

we may conclude that $B^{-1} A^{-1}$ is the inverse of $A B$ 


$$
(A B)^{-1} \equiv B^{-1} A^{-1}
$$

\subsection{Transform of a Product of Operators}

Let $U$ be an operator and $U^{-1}$ its inverse. The transform $A^{\prime}$ by $U$ of an operator $A$ is defined by the identity

$$
A^{\prime} \equiv U A U^{-1}
$$

From the definition of inverse operators () we may write

$$
U A U^{-1} U B U^{-1} f(x)=U A\left(B U^{-1} f(x)+U^{-1} 0\right)=U A B U^{-1} f(x)+U A U^{-1} 0
$$

i.e.

$$
A^{\prime} B^{\prime} f(x)=A^{\prime} B^{\prime}(f(x)+0)=A^{\prime} B^{\prime} f(x)+A^{\prime} B^{\prime} 0=(A B)^{\prime} f(x)+A^{\prime} 0
$$

Because

$$
A^{\prime} B^{\prime} 0=A^{\prime}\left(B^{\prime} 0-0\right)=A^{\prime} B^{\prime} 0-A^{\prime} 0
$$

we finally get the formula

$$
(A B)^{\prime} f(x)=A^{\prime} B^{\prime} f(x)+A^{\prime} B^{\prime} 0=A^{\prime} B^{\prime} f(x) \quad \forall f
$$

saying that the transform of a product of operators is the product of the transformed operators.

\section{The Fundamental Identity in Differential Calculus}

3.1 Proof of $[f(D), X] \equiv f^{\prime}(D)$

In a space of functions let $D$ and $X$ be two operators verifying the relation

From (3.1) we may deduce the following

$$
D X \equiv X D+\hat{I}
$$

$$
D^{m} X \equiv X D^{m}+m D^{m-1}, \quad \forall m \in N
$$

simply by the algorithm based on the remark that according to (3.1), for a product of m operators $D$ and one operator $X$, each time $X$ is moved from right to left of a nearby $D$ we must add $D^{m-1}$ to the result, so that after m such moves $D^{m} X$ becomes $X D^{m}$ plus $D^{m-1}$.

Identity (3.2) is valuable also in the case where $\mathrm{m}$ is a positive rational number. Indeed, let $Y$ be an operator defined by

$$
Y^{n} \equiv D^{m}
$$

and suppose that we can find an operator $A(D)$ which depends only in $D$ and such that

$$
Y X \equiv X Y+A(D)
$$

The above algorithm may be utilized and gives

$$
Y^{n} X \equiv X Y^{n}+n A(D) Y^{n-1}
$$

Replace $Y^{n}$ with $D^{m}$ we have

$$
D^{m} X \equiv X D^{m}+n A(D) D^{\frac{m}{n}(n-1)}
$$

Comparing (3.6) with (3.2) we get

$$
n A(D) D^{\frac{m}{n}(n-1)} \equiv m D^{m-1}
$$

so that (3.5) becomes

$$
D^{\frac{m}{n}} X \equiv X D^{\frac{m}{n}}+\frac{m}{n} D^{\frac{m}{n}-1}
$$

From the above identity we get also 


$$
D^{\frac{m}{n}} X D^{-\frac{m}{n}} \equiv X+\frac{m}{n} D^{-1}
$$

$$
X D^{-\frac{m}{n}} \equiv D^{-\frac{m}{n}} X+\frac{m}{n} D^{-\frac{m}{n}-1}
$$

Because a real number is the limit of two series of rational numbers we may assumed that (3.2) is also valuable for $m$ real.

From (3.2) we may conclude that if $f(x)$ is a differentiable function

$$
f(x) \equiv \sum_{m} a_{m} x^{m} \quad \forall m \in \mathfrak{R}
$$

and $f^{\prime}(x)$ is its derivative function

$$
f^{\prime}(x) \equiv \sum_{m} a_{n} m x^{m-1} \quad \forall m \in \Re
$$

we have the identity

$$
\begin{gathered}
f(D) X \equiv X f(D)+f^{\prime}(D) \\
{[f(D), X] \equiv f^{\prime}(D)}
\end{gathered}
$$

which when apply on a function $g(x)$ must respect the primordial remark that

$$
A g(x)=A g(x)+A 0
$$

3.2 Proof of $f(D) X^{m} \equiv \sum_{k=0}^{m}\left(\begin{array}{l}m \\ k\end{array}\right) X^{m-k} f^{(k)}(D)$

From (3.11) we deduce successively that

$$
\begin{aligned}
& f(D) X^{2} \equiv\left(X f(D)+f^{\prime}(D)\right) X \equiv X^{2} f(D)+2 X f^{\prime}(D)+f^{\prime \prime}(D) \\
& f(D) X^{3} \equiv X^{3} f(D)+3 X^{2} f^{\prime}(D)+3 X f^{\prime \prime}(D)+f^{\prime \prime \prime}(D)
\end{aligned}
$$

and so on for $f(D) X^{4}$, etc... Thank to this remark we suppose that

$$
f(D) X^{m} \equiv \sum_{k=0}^{m}\left(\begin{array}{l}
m \\
k
\end{array}\right) X^{m-k} f^{(k)}(D)
$$

In order to prove (3.12) by recursion we utilize (3.11) to proceed

$$
\begin{aligned}
& f(D) X^{m+1} \equiv \sum_{k=0}^{m}\left(\begin{array}{c}
m \\
k
\end{array}\right) X^{m-k} f^{(k)}(D) X \equiv \sum_{k=0}^{m}\left(\begin{array}{c}
m \\
k
\end{array}\right) X^{m-k}\left(X f^{(k)}(D)+f^{(k+1)}(D)\right) \\
& \equiv \sum_{k=0}^{m}\left(\begin{array}{c}
m \\
k
\end{array}\right)\left(X^{m+1-k} f^{(k)}(D)+\sum_{k=1}^{m+1}\left(\begin{array}{c}
m \\
k-1
\end{array}\right) X^{m-k+1} f^{(k)}(D)\right) \\
&\left.\left.\equiv X^{m+1} f(D)+\sum_{k=1}^{m}\left(\begin{array}{c}
m \\
k
\end{array}\right)+\left(\begin{array}{c}
m \\
k-1
\end{array}\right)\right) X^{m-k+1} f^{(k)}(D)\right)+f^{(m+1)}(D)
\end{aligned}
$$

and get

$$
f(D) X^{m+1} \equiv \sum_{k=0}^{m+1}\left(\begin{array}{c}
m+1 \\
k
\end{array}\right) X^{m+1-k} f^{(k)}(D) \quad \text { QED }
$$

Combining the above result and the fact that $f(D) X^{0} \equiv X^{0} f(D)$ we may conclude that (3.12) is correct.

\subsection{The Fundamental Identity in Differential Calculus}

Under the form (3.12) we can't proceed further because the mixed coefficient $(m-k)$ ! doesn't permit summations with respect to $\mathrm{m}$. In order to bypass this obstacle we make use of the relation 


$$
\frac{m !}{(m-\mathrm{k}) !} x^{m-k}=m(m-1) \ldots(m-k+1) x^{m-k} \equiv x^{m(k)}
$$

where $x^{m(k)}$ is the k-order derivative of $x^{m}$ and obtain

$$
f(D) X^{m} \equiv \sum_{k=0}^{m}\left(\begin{array}{l}
m \\
k
\end{array}\right) X^{m-k} f^{(k)}(D) \equiv \sum_{k=0}^{m} \frac{1}{k !} X^{m(k)} f^{(k)}(D) \equiv \sum_{k=0}^{\infty} \frac{1}{k !} X^{m(k)} f^{(k)}(D)
$$

where $X^{m(k)}$ is obtained by replacing $\mathrm{x}$ with the operator $X$ in the function $x^{m(k)}$.

Finally we may conclude that

"Let $f(x)$ be any differentiable function of the argument $\mathrm{x}, g(x)$ any function expandable into Taylor series, $f^{(k)}(x)$ and $g^{(k)}(x)$ respectively the k-order derivative with respect to $\mathrm{x}$ of $f(x)$ and $g(x)$, then

$$
f(D) g(X)=\sum_{k=0}^{\infty} \frac{1}{k !} g^{(k)}(X) f^{(k)}(D) ”
$$

\subsection{Invariance of the Fundamental Identity}

Inspecting the way we obtain the fundamental identity in differential calculus (3.15) we see that it is the consequence of one and only one condition which is that the ordered couple of operators $(D, X)$ respects the basic identity (3.1)

$$
[D, X] \equiv \hat{I}
$$

We thus obtain an extremely important corollary saying that the fundamental identity is invariant under replacing the couple of operators $(D, X)$ with any other couple $(A, B)$ respecting the condition $[A, B] \equiv \hat{I}$.

From this corollary we get the twin identity of the fundamental one. Indeed by remarking that

$$
[-X, D] \equiv-X D+D X \equiv \hat{I}
$$

we get

$$
f(-X) g(D) \equiv \sum_{k=0}^{\infty} \frac{1}{k !} g^{(k)}(D) f^{(k)}(-X)
$$

Putting $h(x)=f(-x)$, we have $h^{\prime}(x)=-f^{\prime}(-x)$ and

$$
h(X) g(D) \equiv \sum_{k=0}^{\infty} \frac{(-)^{k}}{k !} g^{(k)}(D) h^{(k)}(X)
$$

i.e., because $h(x)$ is also arbitrary as is $f(x)$

$$
f(X) g(D) \equiv \sum_{k=0}^{\infty} \frac{(-)^{k}}{k !} g^{(k)}(D) f^{(k)}(X) \quad \text { QED }
$$

By replacing $(D, X)$ with $\left(\alpha a^{+}+\beta a, \gamma a^{+}+\delta a\right)$ where $\alpha \delta-\beta \gamma=1$ or with $\left(\frac{1}{u^{\prime}(x)} D_{x}, u(\hat{X})\right)$ in (3.16) we obtain an infinity of identities for differential calculus.

With the replacement by $\left(D_{x}+u(\hat{X}), \hat{X}\right)$ we get for example

$$
f(D+u(\hat{X})) g(\hat{X})=\sum_{k=0}^{\infty} \frac{1}{k !} g^{(k)}(\hat{X}) f^{(k)}(D+u(\hat{X})) g(\hat{X})
$$

By the way we see that the Newton binomial may be put under the forms

$$
\begin{aligned}
(x+a)^{\mathrm{m}}=\sum_{k=0}^{m}\left(\begin{array}{l}
m \\
k
\end{array}\right) a^{k} x^{m-k}=\sum_{k=0}^{m} \frac{1}{\mathrm{k} !} a^{k} x^{m^{(k)}} \\
=\sum_{k=0}^{m} \frac{1}{\mathrm{k} !} a^{k} D_{x}{ }^{k} x^{m}=e^{a D_{x}} x^{m}
\end{aligned}
$$




\subsection{The Fundamental Identity in Quantum Mechanics}

Consider the couple of operators

$$
\begin{aligned}
& a^{+} \equiv \frac{1}{\sqrt{2}}\left(D_{x}-\hat{X}\right) \\
& a \equiv \frac{1}{\sqrt{2}}\left(D_{x}+\hat{X}\right)
\end{aligned}
$$

which are the creation and annihilation operators in quantum mechanics.

Because

$$
\left[a^{+}, a\right] \equiv \frac{1}{2}\left(\left[D_{x}, \hat{X}\right]-\left[\hat{X}, D_{x}\right]\right) \equiv \hat{I}
$$

we get according to the invariance property of the identity (3.15)

$$
\begin{gathered}
f\left(a^{+}\right) g(a) \equiv \sum_{k=0}^{\infty} \frac{1}{k !} g^{(k)}(a) f^{(k)}\left(a^{+}\right) \\
f(a) g\left(a^{+}\right) \equiv \sum_{k=0}^{\infty}(-)^{k} \frac{1}{k !} g^{(k)}\left(a^{+}\right) f^{(k)}(a)
\end{gathered}
$$

and similar identities corresponding to the replacements of $\left(a^{+}, a\right)$ with couples $\left(\alpha a^{+}+\beta a, \gamma a^{+}+\delta a\right)$ where $\alpha \delta-\beta \gamma=1$.

\section{Applications}

\subsection{Calculation of primitives and derivatives}

(i) Derivative of a product of two functions

Apply the fundamental identity on a function $h(x)$ we find again the formula

$$
f\left(D_{x}\right) g(x) h(x)=\sum_{k=0}^{\infty} \frac{1}{k !} g^{(k)}(x) f^{(k)}\left(D_{x}\right) h(x)
$$

that Forsyth (1888) had found by generalizing the Leibnitz formula but did not give details of calculation.

An example is

$$
D_{x}^{4} \sin x x^{4}=(\sin x) 4 !+\frac{1}{1 !}(\cos x) 24 x+\frac{1}{2 !}(-\sin x) 12 x^{2}+\frac{1}{3 !}(-\cos x) 4 x^{3}+\frac{1}{4 !}(\sin x) x^{4}
$$

(ii) Change the primitive of $u v^{(n)}$ into that of $v u^{(n)}$

With $f\left(D_{x}\right) \equiv D_{x}^{-1} \equiv \int$ we get from (3.15)

$$
\begin{aligned}
\int u v^{(n)} & =D_{x}^{-1} u D_{x}^{n} v=D_{x}^{-1}\left(D_{x}^{n} u-n D_{x}^{n-1} u^{\prime}+\ldots+(-)^{n} u^{(n)}\right) v \\
& =D_{x}^{n-1} u v-n D_{x}^{n-2} u^{\prime} v+\ldots+(-)^{n-1} n u v+(-)^{n} \int u^{(n)} v
\end{aligned}
$$

(iii) n-order primitive of a function

Utilizing the formula

$$
\int u v^{\prime}=u v-\int u^{\prime} v, \quad \forall v
$$

and putting $v=\int v^{\prime}$ we get the formula

$$
\int u v^{\prime}=u \int v^{\prime}-\int u^{\prime} \int v^{\prime}, \quad \forall v
$$

which leads to the identity 


$$
\int u \equiv u \int-\int u^{\prime} \int
$$

Applying the operator $\int$ onto both members of (4.3) and reutilizing (4.3) we get

$$
\iint u \equiv\left(u \int-\int u^{\prime} \int\right) \int-\iint u^{\prime} \int
$$

Defining

$$
\int^{n} \equiv D_{x}^{-n}
$$

we may write

$$
\int^{2} u \equiv u \int^{2}-\int^{1} u^{\prime} \int^{2}-\int^{2} u^{\prime} \int^{1}
$$

By recursion we obtain the identity

$$
\int^{n} u \equiv u \int^{n}-\int^{1} u^{\prime} \int^{n}-\int^{2} u^{\prime} \int^{n-1}-\ldots-\int^{n} u^{\prime} \int^{1}
$$

Applying this identity onto a function $P(x)$ we get the formula

$$
\int^{n} u P(x)=u \int^{n} P(x)-\sum_{k=1}^{n} \int^{k} u^{\prime} \int^{n-k+1} P(x)+\int^{n} 0
$$

(iv) Primitive of functions containing $\ln \mathrm{x}$

Putting $u=\ln x$ in (4.6) we get

$$
\int^{n} \ln \hat{X} \equiv \ln \hat{X} \int^{n}-\sum_{k=1}^{n} \int^{k} \hat{X}^{-1} \int^{n-k+1}
$$

and

$$
\int^{n} \ln x P(x)=\ln x \int^{n} P(x)-\sum_{k=1}^{n} \int^{k} \frac{1}{x} \int^{n-k+1} P(x)+\int^{n} 0
$$

With $P(x)=1$ we have the formula

$$
\int^{n} \ln x=\frac{x^{n}}{n !}\left(\ln x-\left(1+\frac{1}{2}+\ldots+\frac{1}{n}\right)\right)+q_{n-1}(x)+r_{n-1}(x) \ln x
$$

allowing the calculation of the n-order primitive of $\ln x$ and from that of $(x+a)^{-m}, P_{n}^{-m}(x),(\ln x)^{2}$, etc....

For examples

$$
\begin{aligned}
& \quad \iint \ln x=\frac{x^{2}}{2} \ln x-\frac{3}{4} x^{2}+q_{1}(x)+r_{1}(x) \ln x \\
& \circ \quad \int x \ln x=\ln x \int x-\int \frac{1}{x} \int x=\frac{x^{2}}{2} \ln x-\frac{x^{2}}{4}+q_{0}+r_{0} \ln x \\
& \circ \quad \iint \ln x \ln x=\ln x \iint \ln x-\int \frac{1}{x} \iint \ln x-\iint \frac{1}{x} \int \ln x+q_{1}(x)+r_{1}(x) \ln x \\
& =\frac{1}{2} x^{2} \ln ^{2} x-\frac{3}{2} x^{2} \ln x+\frac{7}{4} x^{2}+q_{1}(x)+r_{1}(x) \ln x
\end{aligned}
$$

4.2 Resolution of differential equations with constant coefficients

(i) Equation $\left(D_{x}-\alpha\right)^{k} y=0$

From the fundamental identity we get for all $\mathrm{j}<\mathrm{k}$ 


$$
\left(D_{x}-\alpha\right)^{k} \hat{X}^{j} \equiv \hat{X}^{j}\left(D_{x}-\alpha\right)^{k}+\frac{j k}{1 !} \hat{X}^{j-1}\left(D_{x}-\alpha\right)^{k-1}+\ldots+\frac{k !}{(k-j) !}\left(D_{x}-\alpha\right)^{k-j}
$$

so that

$$
\left(D_{x}-\alpha\right)^{k} x^{j} e^{\alpha x}=0 \quad \forall j<k
$$

and that the solution of the differential equation

$$
\left(D_{x}-\alpha\right)^{k} y_{k}=0
$$

is

$$
y_{k}=Q_{k-1}(x) e^{\alpha x} \quad \forall Q_{k-1}(x), \text { polynomial order }(k-1)
$$

For generalization consider the equation with constant coefficients

$$
a_{n} y^{(n)}+a_{n-1} y^{(n-1)}+\ldots+a_{1} y^{\prime}+y=0
$$

which may be written under the form

$$
\begin{aligned}
P_{n}\left(D_{x}\right) y & =\left(a_{n} D_{x}^{n}+a_{n-1} D_{x}^{n-1}+\ldots+a_{1} D_{x}+a_{0}\right) y \\
& =a_{n}\left(D_{x}-\alpha_{1}\right)^{k_{1}}\left(D_{x}-\alpha_{2}\right)^{k_{2}} \ldots . .\left(D_{x}-\alpha_{m}\right)^{k_{m}} y=0
\end{aligned}
$$

where $\alpha_{j}$ denotes the root with multiplicity $k_{j}$ of the characteristic polynomial $P_{n}(x)$.

Because the operators $\left(D_{x}-\alpha_{j}\right)^{k_{j}}$ commute one another, according to (4.11) the solution of $P_{n}\left(D_{x}\right) y=0$ is

$$
y=\sum_{j=1}^{m} Q_{k_{j}-1}(x) e^{\alpha_{j} x}, \quad \forall Q_{k_{j}-1}(x)
$$

(ii) Equations with eigenfunction in second member

$$
(A-\lambda)^{m} y=V(x) \text { where } A V(x)=\lambda V(x)
$$

Consider an arbitrary function $f(x)$. By the fundamental identity (3.15) we have

$$
f^{m}\left(D_{x}\right) \hat{X}^{m} \equiv \hat{X}^{m} f^{m}\left(D_{x}\right)+\ldots+\frac{1}{k !}\left(\hat{X}^{m}\right)^{(k)}\left(f^{m}\left(D_{x}\right)\right)^{(k)}+\ldots+\frac{m !}{m !}\left(f^{m}\left(D_{x}\right)\right)^{(m)}
$$

Searching for a term which doesn't contain $\mathrm{f}\left(D_{x}\right)$ in this identity, we see that all the derivatives of order $\mathrm{k}=1$, $2, \ldots, \mathrm{m}-1$ of $\mathrm{f}^{m}(x)$ contain $\mathrm{f}(x)$ and in $\left(\mathrm{f}^{m}(x)\right)^{(m)}$ only the term $m !\left(f^{\prime}(x)\right)^{m}$ doesn't contain $\mathrm{f}(x)$.

By these remarks we see that when applying both members of the previous identity on a function $\mathrm{V}(x)$ verifying the property $f\left(D_{x}\right) V(x)=0$ we have

$$
f^{m}\left(D_{x}\right) x^{m} V(x)=m !\left(f^{\prime}\left(D_{x}\right)\right)^{m} V(x)
$$

Applying the above remark for $(A-\lambda \hat{I})^{m}$ where $(A-\lambda \hat{I}) V(x)=0$ we get the important formula

$$
(A-\lambda)^{m} x^{m} V(x)=m !\left(A^{\prime}\right)^{m} V(x)
$$

and by applying $(A-\lambda \hat{I})^{k}$ on both sides

$$
(A-\lambda)^{m+k} x^{m} V(x)=0 \quad \forall k>0
$$

These formulae lead to the proposition 
« The differential equation

$$
(A-\lambda)^{m} y=V(x)
$$

where

$$
A V(x)=\lambda V(x)
$$

has as general solution

$$
y=\frac{1}{m !\left(A^{\prime}\left(D_{x}\right)\right)^{m}} x^{m} V(x)+\left(c_{1} x^{m-1}+\ldots+c_{m}\right) V(x) \quad \forall c_{i} \quad »
$$

In the case where

$$
m !\left(A^{\prime}\left(D_{x}\right)\right)^{m} V(x)=\alpha_{m} V(x)
$$

the solution is according to (4.15)

$$
y=\frac{1}{\alpha_{m}} x^{m} V(x)
$$

Generalizing for the equation

$$
(A-\lambda I)^{m} y=\sum_{i=1}^{n} a_{i} V_{i}(x) \text { where } A V_{i}(x)=\lambda V_{i}(x)
$$

we see that if we can find a funtion $\sum_{i=1}^{n} \alpha_{i} V_{i}(x)$ so that

$$
m !\left(A^{\prime}\right)^{m} \sum_{i=1}^{n} \alpha_{i} V_{i}(x)=\sum_{i=1}^{n} a_{i} V_{i}(x)
$$

then from (4.15)

$$
(A-\lambda I)^{m} x^{m} \sum_{i=1}^{n} \alpha_{i} V_{i}(x)=m !\left(A^{\prime}\right)^{m} \sum_{i=1}^{n} \alpha_{i} V_{i}(x)=\sum_{i=1}^{n} a_{i} V_{i}(x)
$$

we get the solution

$$
y_{p}=x^{m} \sum_{i=1}^{n} \alpha_{i} V_{i}(x)
$$

By the way we would like to highlight the formula

$$
\frac{1}{(A-\lambda I)^{m}} V(x)=\frac{1}{m !\left(A^{\prime}\right)^{m}} x^{m} V(x)
$$

which is to be add to the well known formula of Cayley concerning the eigenfunction and eigenvalue of an operator

$$
\circ \quad f(A) V(x)=f(\lambda) V(x)
$$

where $f(x)$ is expandable in Taylor series.

(iii) Differential equations $P_{n}\left(D_{x}\right) y=e^{\omega x}$

From the hereabove proposition we see that the differential equation

$$
\left(D_{x}-\omega\right)^{m} y=e^{\omega x}
$$

corresponds to $A\left(D_{x}\right) \equiv\left(D_{x}-\omega\right)$ and $A^{\prime}\left(D_{x}\right) \equiv \hat{I}$ so that the solution is

$$
y=\frac{1}{m !} x^{m} e^{\omega x}+\left(c_{1} x^{m-1}+\ldots+c_{m}\right) e^{\omega x} \quad \forall c_{i}
$$

Generalizing for the equation 


$$
P_{n}\left(D_{x}\right) y=Q\left(D_{x}\right)\left(D_{x}-\omega\right)^{m} y=e^{\omega x}
$$

where $\omega$ is the root of multiplicity $m \geq 0$ of the characteristic polynomial we see that

$$
Q\left(D_{x}\right)\left(D_{x}-\omega\right)^{m} x^{m} e^{\pi x}=Q\left(D_{x}\right) m !(1)^{m} e^{\omega x}=Q(\omega) m ! e^{\omega x}
$$

The general solution is then

$$
y=\frac{1}{m ! Q(\omega)} x^{m} e^{\omega x}+\left(c_{1} x^{m-1}+\ldots+c_{m}\right) e^{\omega x} \quad \forall c_{i}
$$

(iv) Differential equations with sinusoidal functions at second member

$$
P_{n}\left(D_{x}\right) y=a \cos \omega x+b \sin \omega x
$$

If $P_{n}\left(D_{x}\right)$ has $i \omega$ as root of multiplicity $m \geq 0$ and $(-i \omega)$ as root of multiplicity $n \geq m$ or vice versa we may write

$$
P_{n}\left(D_{x}\right) \equiv Q\left(D_{x}\right)\left(D_{x}^{2}+\omega^{2}\right)^{m}
$$

Because

$$
\left(D_{x}^{2}+\omega^{2}\right)^{m}(\alpha \cos \omega \mathrm{x}+\beta \sin \omega \mathrm{x})=0 \quad \forall \alpha, \beta
$$

we have according to (4.15) with $A\left(D_{x}\right) \equiv\left(D_{x}{ }^{2}+\omega^{2}\right), A^{\prime}\left(D_{x}\right) \equiv 2 D_{x}$

$$
\begin{aligned}
& \left(D_{x}^{2}+\omega^{2}\right)^{m} \hat{X}^{m}(\alpha \cos \omega x+\beta \sin \omega x)=m !\left(2 D_{x}\right)^{m}(\alpha \cos \omega x+\beta \sin \omega x) \\
& P_{n}\left(D_{x}\right) \hat{X}^{m}(\alpha \cos \omega x+\beta \sin \omega x)=Q\left(D_{x}\right) m !\left(2 D_{x}\right)^{m}(\alpha \cos \omega x+\beta \sin \omega x)
\end{aligned}
$$

Choosing $\alpha, \beta$ so that

$$
Q\left(D_{x}\right) m !\left(2 D_{x}\right)^{m}(\alpha \cos \omega x+\beta \sin \omega x)=(a \cos \omega x+b \sin \omega x)
$$

which is a very easy problem because

$$
D_{x}^{2 n}(\alpha \cos \omega x+\beta \sin \omega x)=(-)^{n} \omega^{2 n}(\alpha \cos \omega x+\beta \sin \omega x)
$$

we have the proposition

« The general solution of the equation

$$
P_{n}\left(D_{x}\right) y=Q\left(D_{x}\right)\left(D_{x}^{2}+\omega^{2}\right)^{m} y=(a \cos \omega x+b \sin \omega x)
$$

is

$$
y(x)=\left(x^{m}+c_{1} x^{m-1}+\ldots+c_{m}\right)(\alpha \cos \omega x+\beta \sin \omega x) \forall c_{i}
$$

where $(\alpha \cos \omega x+\beta \sin \omega x)$ is given by the equation

$$
Q\left(D_{x}\right) m !\left(2 D_{x}\right)^{m}(\alpha \cos \omega x+\beta \sin \omega x)=(a \cos \omega x+b \sin \omega x) "
$$

The above equation for finding the trial solution $(\alpha \cos \omega x+\beta \sin \omega x)$ is certainly far easier to perform than the trial equation

$$
P_{n}\left(D_{x}\right) x^{m}(\alpha \cos \omega x+\beta \sin \omega x)=(a \cos \omega x+b \sin \omega x)
$$

teached in nowadays textbooks. 
For example consider the equation

$$
P_{n}\left(D_{x}\right) y=\left(D_{x}^{7}+D_{x}^{4}+1\right)\left(D_{x}^{2}+4\right)^{3} y=\cos 2 x+3 \sin 2 x
$$

We search $\alpha, \beta$ by identification of coefficients of $\cos 2 x$ and $\sin 2 x$ in

$$
\left(D_{x}^{7}+D_{x}^{4}+1\right) 3 !\left(2 D_{x}\right)^{3}(\alpha \cos 2 x+\beta \sin 2 x)=\cos 2 x+3 \sin 2 x
$$

The details of calculation are

$$
\begin{aligned}
& \left(D_{x}^{10}+D_{x}^{7}+D_{x}^{3}\right) 48(\alpha \cos 2 x+\beta \sin 2 x)=\cos 2 x+3 \sin 2 x \\
& \left((-4)^{5}+(-4)^{3} D_{x}+(-4)^{1} D_{x}\right) 48(\alpha \cos 2 x+\beta \sin 2 x)=\cos 2 x+3 \sin 2 x \\
& \left(-1024-68 D_{x}\right)(\alpha \cos 2 x+\beta \sin 2 x)=\frac{1}{48}(\cos 2 x+3 \sin 2 x) \\
& -256 \alpha-34 \beta=\frac{1}{192}, \quad-256 \beta+34 \alpha=\frac{3}{192}
\end{aligned}
$$

Once $\alpha, \beta$ calculated, the particular solution is

$$
y_{p}(x)=x^{3}(\alpha \cos 2 x+\beta \sin 2 x)
$$

(v) Differential equations with polynomials at second member

$$
P_{n}\left(D_{x}\right) y=T_{m}(x)
$$

Consider the equation

$$
P_{n}\left(D_{x}\right) y=\left(a_{n} D_{x}^{n}+\ldots+a_{1} D_{x}+a_{0}\right) y=T_{m}(x), a_{0} \neq 0
$$

Applying $D_{x}^{m+1}$ on both sides we have

$$
P_{n}\left(D_{x}\right) D_{x}^{m+1} y=D_{x}^{m+1} T_{m}(x)=0
$$

and see that $D_{x}{ }^{m+1} y=0$ i.e. y must be a polynomial of order $\mathrm{m}$.

Now, let us introduce a polynomial $Q_{m}\left(D_{x}\right)$ such that

$$
Q_{m}\left(D_{x}\right) P_{n}\left(D_{x}\right) \equiv 1+0+\ldots+0+r_{m+1} D_{x}^{m+1}+r_{m+2} D_{x}^{m+2}+\ldots .
$$

we obtain immediately a particular solution $y_{p}$ of the equation because

$$
Q_{m}\left(D_{x}\right) P_{n}\left(D_{x}\right) y_{p}=y_{p}=Q_{m}\left(D_{x}\right) T_{m}(x)
$$

To resume we may assert that

"The particular solution of the differential equation

$$
P_{n}\left(D_{x}\right) y=\left(a_{n} D_{x}{ }^{n}+\ldots+a_{1} D_{x}+a_{0}\right) y=T_{m}(x), a_{0} \neq 0
$$

is

$$
y_{p}=Q_{m}\left(D_{x}\right) T_{m}(x)
$$

where the coefficients $q_{i}$ of the polynomial $Q_{m}\left(D_{x}\right)$ verify the system of algebraic equations

$$
\begin{aligned}
q_{0} a_{0} & =1 \\
q_{0} a_{i}+q_{1} a_{i-1}+\ldots+q_{i} a_{0} & =0, \quad i=1,2, \ldots, m ”
\end{aligned}
$$


For example for

$$
P_{n}(D) y=\left(D^{4}+5 D^{3}-2 D^{2}+4 D+1\right) y=T_{m}(x)
$$

we have

$$
\begin{aligned}
& q_{0} \cdot 1=1 \Rightarrow q_{0}=1 \\
& 4 q_{0}+q_{1}=0 \Rightarrow q_{1}=-4 \\
& -2 q_{0}+4 q_{1}+q_{2}=0 \Rightarrow q_{2}=18 \\
& 5 q_{0}-2 q_{1}+4 q_{2}+q_{3}=0 \Rightarrow q_{3}=-85 \\
& q_{0}+5 q_{1}-2 q_{2}+4 q_{3}+q_{4}=0 \Rightarrow q_{4}=395
\end{aligned}
$$

so that

$$
y_{p}=\left(1-4 D+18 D^{2}-85 D^{3}+395 D^{4}\right) T_{m}(x)
$$

The present method for obtaining

$$
y_{p}=Q_{m}\left(D_{x}\right) T_{m}(x)
$$

is convenient because $Q_{m}\left(D_{x}\right)$ is easy to get and doesn't depend on the second member $T_{m}(x)$.

(vi) Equations utilizing Heaviside function and Dirac delta function

$$
\left(D^{2}-a^{2}\right) y=e^{b|x|}
$$

Utilizing the function Dirac delta function $\delta(x)$ having the special property $x \delta(x)=0$ and the Heaviside step function $H(x)=0$ for $x<0, H(x)=1$ for $x>0$, we have

$$
\begin{aligned}
& \operatorname{sgn}(x)=H(x)-H(-x), H^{\prime}(x)=\delta(x), \operatorname{sgn}^{\prime}(x)=2 \delta(x) \\
& D_{x} e^{a|x|}=e^{a x \operatorname{sgn}(x)}=a(\operatorname{sgn}(x)+2 x \delta(x)) e^{a|x|}=\operatorname{a~sgnx} e^{a|x|}
\end{aligned}
$$

From these formulae we get

$$
\begin{aligned}
& \left(D_{x}^{2}-a^{2}\right) e^{a|x|}=2 a \delta(x) e^{a|x|}=2 a \delta(x) \\
& \left.\left(D_{x}^{2}-a^{2}\right) e^{b|\hat{X}|}=\left(D_{x}^{2}-b^{2}-a^{2}+b^{2}\right) e^{b|\hat{X}|}\right)=2 b \delta(x)+\left(b^{2}-a^{2}\right) e^{b|\hat{X}|}
\end{aligned}
$$

Combining these two formulae so that $\delta(x)$ disappears we may conclude that

"The general solution of the equation

$$
\left(D^{2}-a^{2}\right) y=e^{b|x|}, \quad a \neq b
$$

is

$$
y=\frac{1}{a\left(a^{2}-b^{2}\right)}\left(b e^{a|x|}-a e^{b|x|}\right)+\left(D^{2}-a^{2}\right)^{-1} 0
$$

or

$$
y=\frac{1}{\left(D_{x}^{2}-a^{2}\right)} e^{b|x|}=y_{p}+c_{1} \cosh a x+c_{2} \sinh a x \quad \forall c_{1}, c_{2}{ }^{\prime}
$$

From the above result we may resolve the equations

$$
P_{n}\left(D_{x}^{2}\right) y=e^{b|x|}
$$

and, remarking that $Q_{n}\left(-D_{x}\right) Q_{n}\left(D_{x}\right)$ is a function of $D_{x}^{2}$, some of the equations of the form $Q_{n}\left(D_{x}\right) y=e^{b|x|}$. 
(vii) Equations with arbitrary second member

$$
\left(D_{x}-a\right)^{m} y=f(x)
$$

From the fundamental identity we may factorize the $\operatorname{sum}\left(D_{x}-a \hat{I}\right)$

$$
e^{a x} D_{x} e^{-a x} \equiv D_{x}-a \hat{I}
$$

and get its inverse

$$
\left(D_{x}-a \hat{I}\right)^{-1} \equiv e^{a x} \int e^{-a x}
$$

More generally

$$
\left(D_{x}-a \hat{I}\right)^{-m} \equiv e^{a x} \int^{m} e^{-a x}
$$

The above identity allows us to conclude that

"The solution of the equation

$$
\left(D_{x}-a\right)^{m} y=f(x)
$$

is

$$
y=e^{a x} D_{x}^{-m} e^{-a x} f(x)=e^{a x} \int^{m} e^{-a x}(f(x)+0) ”
$$

Generalizing to equations

$$
P_{n}(D) y=a_{n}\left(D-\alpha_{n}\right)\left(D-\alpha_{n-1}\right) \ldots . .\left(D-\alpha_{1}\right) y=f(x) \quad \alpha_{i} \leq \alpha_{i+1}
$$

we have the solution

$$
y=e^{\alpha_{n} x} \int e^{\left(-\alpha_{n}+\alpha_{n-1}\right) x} \int \ldots \int e^{\left(-\alpha_{2}+\alpha_{1}\right) x} \int e^{-\alpha_{1} x} f(x) d x
$$

\subsection{Resolution of differential equations of $1^{\text {st }}$ order}

Consider the equation

$$
y^{\prime}+a(x) y=\left(D_{x}+a(x)\right) y=f(x)
$$

By the fundamental identity (3.15) we have

$$
D_{x} e^{A(\hat{X})} \equiv e^{A(\hat{X})} D_{x}+A^{\prime}(\hat{X}) e^{A(\hat{X})}
$$

so that

$$
D_{x}+A^{\prime}(\hat{X}) \equiv e^{-A(\hat{X})} D_{x} e^{A(\hat{X})}
$$

Acccording to the above formula we see that

"The solution of the equation

$$
y^{\prime}+a(x) y=\left(D_{x}+a(x)\right) y=f(x)
$$

is, with $A(x)=\int a(x)$,

$$
y=e^{-A(x)} \int e^{A(x)} f(x)+e^{-A(x)} \int 0 ”
$$

\subsection{Solution of Second Order Differential Equations}

Consider the equation 


$$
\left(D_{x}+a(x)\right)\left(D_{x}+b(x)\right) y=f(x)
$$

From (4.40) we may write this equation under the form

$$
e^{-A(x)} D_{x} e^{A(x)-B(x)} D_{x} e^{B(x)} y=f(x)
$$

where $\mathrm{A}(x)$ and $\mathrm{B}(x)$ are respectively primitive of $\mathrm{a}(x)$ and $\mathrm{b}(x)$.

As consequence we get the proposition

"The general solution of the equation

$$
\left(D_{x}+a(x)\right)\left(D_{x}+b(x)\right) y=f(x)
$$

or

$$
\left(D_{x}^{2}+(a+b) D_{x}+a b+b^{\prime}\right) y=f(x)
$$

is

$$
y=e^{-B(x)} \int e^{B(x)-A(x)} \int e^{A(x)}(f(x)+0) ”
$$

For example, the solution of the equation

$$
(D-\operatorname{tg} x)(D+\operatorname{tg} x) y=\operatorname{tg} x
$$

is

$$
\begin{aligned}
& y=\cos x \int \frac{1}{\cos ^{2} x} \int \cos x \operatorname{tg} x=-\cos x \int \frac{\cos x}{1-\sin ^{2} x} \\
& y_{p}=-\frac{1}{2} \cos x \ln \frac{1+\sin x}{1-\sin x}=-\cos x \ln \left|\frac{1+\sin x}{\cos x}\right|
\end{aligned}
$$

4.5 Resolution of Differential Equations by Differential Transformation

(i) Consider the equation

$$
P\left(D_{x}, \hat{X}\right) y=f(x)
$$

Applying on both sides by an operator $A\left(D_{x}\right)$ or $A(\hat{X})$ and utilizing the identities deduced from the fundamental one

$$
\begin{aligned}
& A\left(D_{x}\right) P\left(D_{x}, \hat{X}\right) \equiv P A+\ldots+\frac{1}{k !} P_{X}{ }^{(k)} A^{(k)}+\ldots, P_{X}{ }^{(k)} \equiv \partial_{\hat{X}}{ }^{k} P\left(\hat{X}, D_{x}\right) \\
& A(\hat{X}) P\left(D_{x}, \hat{X}\right) \equiv P A+\ldots+\frac{1}{k !} P_{D}^{(k)} A^{(k)}+\ldots \quad, P_{D}{ }^{(k)} \equiv \partial_{D_{x}}^{k} P\left(\hat{X}, D_{x}\right)
\end{aligned}
$$

we get the transformed equation

$$
A P\left(D_{x}, \hat{X}\right) y=A f(x)
$$

which may be resolved if the parameters in $A$ are well choosen.

As examples we have

$$
\begin{aligned}
& e^{a \hat{X}^{2}} P\left(D_{x}, \hat{X}\right) \equiv P\left(D_{x}-2 a \hat{X}, \hat{X}\right) e^{a \hat{X}^{2}} \\
& e^{a D_{x}^{2}} P\left(D_{x}, \hat{X}\right) \equiv P\left(D_{x}, \hat{X}+2 a D_{x}\right) e^{a D_{x}^{2}}
\end{aligned}
$$


(ii) Transform of equation

$$
P_{n}\left(D_{x}, \hat{X}\right) y=e^{a x} f(x)
$$

Applying $e^{-a \hat{X}}$ on both sides we transform the equation

$$
P_{n}\left(D_{x}, \hat{X}\right) y=e^{a x} f(x)
$$

into

$$
e^{-a x} P_{n}\left(D_{x}, \hat{X}\right) y=P_{n}\left(D_{x}+a, \hat{X}\right) e^{-a x} y=f(x)
$$

which is an equation for calculating $Y=e^{-a x} y$.

The above method may be applied for other forms of second member.

4.6 Differential calculus for studying Special functions

(i) The Hermite polynomials

Consider the differential equation

$$
\left(D_{x}^{2}-2 x D_{x}+2 n\right) y=0
$$

Let $A$ be an operator depending only on $D_{x}$ we get according to (3.15)

$$
A\left(D_{x}^{2}-2 x D_{x}+2 n\right) y=\left(D_{x}^{2}-2\left(\hat{X}+A^{\prime} A^{-1}\right) D_{x}+2 n\right) A y=0
$$

In order to cancel terms in $D_{x}^{2}$ we make the choice $2 A^{\prime} A^{-1} \equiv D_{x}$ i.e.

$$
\frac{A^{\prime}}{A} \equiv \frac{1}{2} D_{x} \Rightarrow A \equiv e^{\frac{1}{4} D_{x}^{2}}
$$

and get the first order equation

$$
e^{\frac{1}{4} D_{x}^{2}}\left(D_{x}^{2}-2 \hat{X} D_{x}+2 n\right) y=-2\left(x D_{x}-n\right) e^{\frac{1}{4} D_{x}^{2}} y=0
$$

which, because $\left(x D_{x}-n\right) x^{n}=0$, has as solution

$$
y_{n}=c_{n} e^{-\frac{1}{4} D_{x}^{2}} x^{n}
$$

with

$$
y_{2 n}(0)=c_{2 n}(-)^{n} \frac{1}{n ! 2^{2 n}} D_{x}^{2 n} x^{2 n}=(-)^{n} \frac{(2 n-1) ! !}{2^{n}} c_{2 n}
$$

The Hermite polynomial $H_{n}(x)$ is a solution having the property

$$
H_{2 n}(0)=(-)^{n} 2^{n}(2 n-1) ! !, \quad H_{2 n+1}(0)=0
$$

so that

$$
H_{n}(x)=e^{-\frac{1}{4} D_{x}^{2}}(2 x)^{n}
$$

Because according to the fundamental identity (3.15)

$$
2 \hat{X}-D_{x} \equiv e^{-\frac{1}{4} D_{x}^{2}}(2 \hat{X}) e^{-\frac{1}{4} D_{x}^{2}} \equiv-e^{\hat{X}^{2}} D_{x} e^{-\hat{X}^{2}}
$$

we get the Rodrigues formula 
○ $H_{n}(x)=e^{-\frac{1}{4} D_{x}{ }^{2}}(2 x)^{n}=e^{-\frac{1}{4} D_{x}{ }^{2}}(2 x)^{n} e^{\frac{1}{4} D_{x}{ }^{2}} 1=(-)^{n} e^{x^{2}} D_{x}^{n} e^{-x^{2}}$

The generating functions and the main properties of Hermite polynomials may be obtained in a concise manner as we can see hereafter.

$$
\begin{aligned}
& \circ \sum_{n=0}^{n} H_{n}(x) \frac{t^{n}}{n !}=e^{-\frac{D_{x}^{2}}{4}} e^{2 t x}=e^{-t^{2}+2 t x} \\
& \circ \sum_{n-0}^{n} H_{n}(x) H_{n}(y) \frac{t^{n}}{n !}=e^{-\frac{D_{x}^{2}}{4}} e^{-\frac{D_{y}^{2}}{4}} e^{4 x y t}=e^{-\frac{D_{x}^{2}}{4}} e^{-4 x^{2} t^{2}+4 x y t} \\
&=e^{-\frac{D_{x}^{2}}{4}} e^{-4 t^{2}\left(x-\frac{y}{2 t}\right)^{2}+y^{2}}=\left(1-4 t^{2}\right)^{-\frac{1}{2}} e^{y^{2}-\frac{(2 x t-y)^{2}}{1-4 t^{2}}}
\end{aligned}
$$

For proving the above formula we base on the followed remark and calculations

$$
\begin{aligned}
& \left(D_{x}-2 \alpha x\right) e^{\alpha x^{2}}=0 \\
& e^{\beta D_{x}^{2}}\left(D_{x}-2 \alpha \hat{X}\right) e^{\alpha x^{2}}=\left(D_{x}-2 \alpha \hat{X}-4 \alpha \beta D_{x}\right) e^{\beta D_{x}^{2}} e^{\alpha x^{2}}=0
\end{aligned}
$$

to get

$$
e^{\beta D_{x}^{2}} e^{\alpha x^{2}}=c e^{\alpha x^{2} /(1-4 \alpha \beta)}
$$

and after egalization of the constants in both sides

$$
\sum_{n=0}^{\infty} \frac{\alpha^{n} \beta^{n}(2 n) !}{n ! n !}=(1-4 \alpha \beta)^{-\frac{1}{2}}=c
$$

The addition formula for calculating $H_{n}(x+y)$ may be proved by utilizing the differential representation and the remarks that

$$
\begin{gathered}
H_{n}(\alpha x)=e^{-\frac{1}{4 \alpha^{2}} D_{x}^{2}}(2 \alpha x)^{n} \\
D_{x} f(x+y)=D_{y} f(x+y) \\
D_{x}{ }^{n} f(x+y)=D_{x}{ }^{k} D_{y}{ }^{n-k} f(x+y) \quad \forall k \leq n
\end{gathered}
$$

which lead to for example for $\alpha=\sqrt{2}, k=n / 2$

$$
\text { ○ } H_{n}(x+y)=2^{-\frac{n}{2}} \sum_{k=0}^{n}\left(\begin{array}{l}
n \\
k
\end{array}\right) H_{k}(\sqrt{2} x) H_{n-k}(\sqrt{2} y)
$$

By the same manner we may obtain similar formulae for $H_{n}(x+y+\ldots+t)$.

The Hermite polynomials are related to the creation and annihilation operators in quantum mechanics

$$
a^{+} \equiv \frac{1}{\sqrt{2}}\left(D_{x}-\hat{X}\right), a \equiv \frac{1}{\sqrt{2}}\left(D_{x}+\hat{X}\right)
$$

Indeed from (4.53) we get the familiar formula

$$
\text { ○ } \quad H_{n+1}(x)=e^{-\frac{1}{4} D_{x}^{2}}(2 x)^{n+1}=2 x H_{n}(x)-2 n H_{n-1}(x)
$$

which allows us to write 


$$
H_{n+1}\left(\frac{\hat{X}+D_{x}}{\sqrt{2}}\right) \equiv 2 \frac{\hat{X}+D_{x}}{\sqrt{2}} H_{n}\left(\frac{\hat{X}+D_{x}}{\sqrt{2}}\right)-2 n H_{n-1}\left(\frac{\hat{X}+D_{x}}{\sqrt{2}}\right)
$$

and deduce by recursion the remarkable identity

$$
\text { ○ } H_{n}(a) \equiv \sum_{k=0}^{n}\left(\begin{array}{l}
n \\
k
\end{array}\right) \hat{X}^{k} D_{x}^{n-k}
$$

Derivations and m-terms recurrence relations of Hermite polynomials are easily obtained by remarking that from the fundamental identities (3.15), (3.16)

$$
\begin{aligned}
& D_{x}^{m} e^{-\frac{1}{4} D_{x}^{2}} \equiv e^{-\frac{1}{4} D_{x}^{2}} D_{x}^{m} \\
& \hat{X}^{m} e^{-\frac{1}{4} D_{x}^{2}} \equiv e^{-\frac{1}{4} D_{x}^{2}} \hat{X}^{m}+e^{-\frac{1}{4} D_{x}^{2}} \frac{D_{x}}{2} k \hat{X}^{k-1}+\ldots+\frac{1}{m !}\left(e^{-\frac{1}{4} D_{x}^{2}}\right)^{(m)} \hat{X}^{m(m)}
\end{aligned}
$$

As simple example we have

$$
\begin{aligned}
& \circ D_{x} H_{n}(x)=2 n e^{-\frac{1}{4} D_{x}^{2}}(2 x)^{n-1}=2 n H_{n-1}(x) \\
& \circ \quad 2 x H_{n}(x)=2 \hat{X} e^{-\frac{1}{4} D_{x}^{2}}(2 x)^{n}=e^{-\frac{1}{4} D_{x}^{2}}\left(2 \hat{X}+D_{x}\right)(2 x)^{n}=H_{n+1}(x)+H_{n}^{\prime}(x) \\
& \int^{-x^{2}} H_{n+1}(x)=\int e^{-x^{2}} 2 x H_{n}(x)-\int e^{-x^{2}} H^{\prime}(x)=-e^{-x^{2}} H_{n}(x) \\
& \circ \int_{0}^{\infty} e^{-x^{2}} H_{n+1}(x)=H_{n}(0) \\
& \circ \int_{-\infty}^{\infty} e^{-x^{2}} H_{n+1}(x)=H_{n}(0)+(-)^{n+1} H_{n}(0)=0 n \geq 0 \\
& \circ \int_{-\infty}^{\infty} e^{-x^{2}} H_{0}(x)=\sqrt{\pi} \\
& \circ \int^{-x^{2}} H_{n}(x) H_{m}(x)=\int e^{-x^{2}} 2 x H_{n-1}(x) H_{m}(x)-\int e^{-x^{2}} H^{\prime}{ }_{n-1}(x) H_{m}(x)=-e^{-x^{2}} H_{n-1}(x) H_{m}(x)+\int e^{-x^{2}} H_{n-1}(x) H_{m}^{\prime}(x) \\
& \quad=-e^{-x^{2}}\left(H_{n-1}(x) H_{m}(x)+H_{n-2}(x) H_{m}^{\prime}(x)+\ldots\right)+\int e^{-x^{2}} H_{n-m}(x) H_{m}{ }^{(m)}(x)
\end{aligned}
$$

Examining all possibilities for $n \geq m$ concerning parity and remarking that $\int_{0}^{\infty} e^{-x^{2}} H_{n}(x)=\frac{1}{2} \sqrt{\pi} \delta_{n 0}$ we get the orthogality of the Hermite polynomials

$$
\text { ○ } \int_{-\infty}^{\infty} e^{-x^{2}} H_{n}(x) H_{m}(x)=m ! 2^{m} \sqrt{\pi} \delta_{n m}
$$

From the recurrence and derivative formulae we may prove by recursion the following identities

$$
\begin{aligned}
\circ \quad\left(D_{x}+2 \hat{X}\right)^{n} & \equiv \sum_{k=0}^{n}\left(\begin{array}{l}
n \\
k
\end{array}\right) D_{x}{ }^{k} H_{n-k}(\hat{X}) \\
\circ \quad\left(D_{x}-2 \hat{X}\right)^{n} & \equiv \sum_{k=0}^{n}(-)^{k}\left(\begin{array}{l}
n \\
k
\end{array}\right) H_{k}(\hat{X}) D_{x}^{n-k}
\end{aligned}
$$

Replacing $\left(D_{x}, \hat{X}\right)$ with $\left(\sqrt{2} D_{x}, \frac{1}{\sqrt{2}} \hat{X}\right)$ and utilizing the Hermite polynomials $H e_{n}(x)$ defined by

$$
H e_{n}(x)=e^{-\frac{1}{4} D_{x}^{2}} x^{n}=2^{-\frac{n}{2}} H_{n}\left(\frac{x}{\sqrt{2}}\right)
$$

we get 


$$
\begin{aligned}
& \circ\left(D_{x}+\hat{X}\right)^{n} \equiv \sum_{k=0}^{n}\left(\begin{array}{l}
n \\
k
\end{array}\right) D_{x}{ }^{k} H e_{n-k}(\hat{X}) \equiv \sum_{k=0}^{n} \frac{1}{k !}\left(D_{x}{ }^{n}\right)^{(k)} H e_{k}(\hat{X}) \\
& \circ \quad\left(D_{x}-\hat{X}\right)^{n} \equiv \sum_{k=0}^{n}(-)^{k} \frac{1}{k !} H e_{k}(\hat{X})\left(D_{x}{ }^{n}\right)^{(k)}
\end{aligned}
$$

From the above identities we obtain also two identities

$$
\begin{aligned}
& \circ f\left(a^{+}\right) \equiv \sum_{k=0}^{\infty} \frac{1}{k !} f^{(k)}\left(\frac{D_{x}}{\sqrt{2}}\right) H e_{k}(\hat{X}) \\
& \circ f(a) \equiv \sum_{k=0}^{\infty}(-)^{k} \frac{1}{k !} H e_{k}(\hat{X}) f^{(k)}\left(2^{-\frac{1}{2}} D_{x}\right)
\end{aligned}
$$

where $f(x)$ is expandable in Taylor series.

These identities may be generalized for multi- dimensional spaces and allow the calculations of $f\left(a^{+}\right) g(x)$ by $f(a) g(x)$.

(ii) The Laguerre polynomials

Consider the differential equation of Laguerre polynomials

$$
\left(\hat{X} D_{x}^{2}+(1-\hat{X}) D_{x}+n\right) y=\left(\hat{X} D_{x}\left(D_{x}-1\right)+D_{x}+n\right) y=0
$$

Applying an operator $A\left(D_{x}\right)$ onto both sides and utilizing the fundamental identity (3.15) we have

$$
\begin{aligned}
& A \hat{X} \equiv \hat{X} A+A^{\prime} \\
& A\left(\hat{X} D_{x}\left(D_{x}-1\right)+D_{x}+n\right) \equiv\left(\hat{X} D_{x}\left(D_{x}-1\right)+D_{x}+n\right) A+A^{\prime} D_{x}\left(D_{x}-1\right)
\end{aligned}
$$

Searching for $A\left(D_{x}\right)$ such that $A^{\prime} D_{x}\left(D_{x}-1\right)$ contains $A$ we have two choices

$$
A \equiv D_{x}^{-m} \text { and } A \equiv\left(D_{x}-1\right)^{-m}
$$

With the second choice we may transform the Laguerre equation into

$$
\begin{aligned}
\left(D_{x}-1\right)^{-m}\left(\hat{X} D_{x}\right. & \left.\left(D_{x}-1\right)+D_{x}+n\right) y \\
& =\left(\left(\hat{X} D_{x}\left(D_{x}-1\right)+D_{x}+n\right)-m D_{x}\right) A \\
& =\left(\left(\hat{X} D_{x}-m+1\right) D_{x}-\left(\hat{X} D_{x}-n\right)\right)\left(D_{x}-1\right)^{-m} y=0
\end{aligned}
$$

Remarking that

$$
\left(\hat{X} D_{x}-n\right) x^{n}=\left(\hat{X} D_{x}-n+1\right) D_{x} x^{n}=0
$$

we choice $m=n$ and see that

$$
\left(D_{x}-1\right)^{-n} y=c_{n} x^{n}
$$

For conclusion, we may state that

"The differential equation $\left(\hat{X} D_{x}^{2}+(1-\hat{X}) D_{x}+2 n\right) y=0$

has as particular solution

$$
y_{n}=c_{n}\left(D_{x}-1\right)^{n} x^{n}
$$

If we define the Laguerre polynomial as a particular solution verifying the condition

$$
L_{n}(0)=1
$$


then

$$
L_{n}(x)=\left(D_{x}-1\right)^{n} \frac{x^{n}}{n !}
$$

By factorizing $\left(D_{x}-1\right)$ we get the Rodrigues formula and vice versa

$$
\text { ○ } L_{n}(x)=e^{x} D_{x}^{n} e^{-x} x^{n} / n \text { ! }
$$

From (4.61) we get the formula for calculating explicitly $L_{n}(x)$

$$
\begin{aligned}
& \circ \begin{aligned}
L_{n}(x)=(-)^{n}\left(1-D_{x}\right)^{n} \frac{x^{n}}{n !} & =\sum_{k=0}^{n}(-)^{n-k} \frac{n !}{k !(n-k) !} D_{x}{ }^{k} \frac{x^{n}}{n !} \\
& =\sum_{k=0}^{n}(-)^{n-k} \frac{n !}{k !(n-k) !(n-k) !} x^{n-k}=\sum_{k=0}^{n}(-)^{k}\left(\begin{array}{l}
n \\
k
\end{array}\right) \frac{x^{k}}{k !}
\end{aligned} \\
& \circ \quad L_{n}{ }^{(k)}(x)=D_{x}{ }^{k} L_{n}(x)=D_{x}{ }^{k}\left(D_{x}-1\right)^{n} \frac{x^{n}}{n !}=\left(D_{x}-1\right)^{k} L_{n-k}(x)
\end{aligned}
$$

Now, let us prove the orthogonality of Laguerre polynomials.

Remarking that

$$
e^{x} \int e^{-x} \equiv\left(D_{x}-1\right)^{-1}
$$

and utilizing the fundamental identity (3.16) we get

$$
\begin{aligned}
\int e^{-x} L_{n}(x) L_{m}(x) & =e^{-x}\left(D_{x}-1\right)^{-1} L_{n}(x)\left(D_{x}-1\right)^{m} \frac{x^{m}}{m !} \\
& =e^{-x}\left(D_{x}-1\right)^{-1} \sum_{k=0}^{n} \frac{(-)^{k}}{k !}\left(D_{x}-1\right)^{m(k)} L_{n}^{(k)}(x) \frac{x^{m}}{m !} \\
& =e^{-x}\left(D_{x}-1\right)^{-1} \sum_{k=0}^{n} \frac{(-)^{k} m !}{k !(m-k) !}\left(D_{x}-1\right)^{m-k} \frac{x^{m}}{m !}\left(D_{x}-1\right)^{k} L_{n-k}(x) \\
& =e^{-x}\left(D_{x}-1\right)^{-1} \sum_{k=0}^{n} \sum_{i=0}^{k} \frac{(-)^{k} m !}{k !(m-k) !}\left(D_{x}-1\right)^{m-k}(-)^{i}\left(D_{x}-1\right)^{k^{(i)}} \frac{x^{m(i)}}{i ! m !} L_{n-k}(x) \\
& =e^{-x}\left(D_{x}-1\right)^{-1} \sum_{k=0}^{n} \sum_{i=0}^{k} \frac{(-)^{k+i} m !}{k !(m-k) !}\left(D_{x}-1\right)^{m-i} \frac{k !}{(k-i) !} \frac{m ! x^{m-i}}{i !(m-i) ! m !} L_{n-k}(x) \\
\int_{0}^{\infty} e^{-x} L_{n}(x) L_{m}(x) & =-\left.\sum_{k=0}^{n} \sum_{i=0}^{k} \frac{(-)^{k+i} m !}{k !(m-k) !(k-i) ! i !(m-i) !}\left(D_{x}-1\right)^{m-i-1} x^{m-i} L_{n-k}(x)\right|_{x=0}
\end{aligned}
$$

Examining all possibilities for $\mathrm{k}$, $\mathrm{i}$ we see that only for $\mathrm{n}=\mathrm{m}=\mathrm{i}=\mathrm{k}$ that we have a non vanishing term and may conclude that

$$
\circ \quad \int_{0}^{\infty} e^{-x} L_{n}(x) L_{m}(x)=-\left(D_{x}-1\right)^{-1} x^{0} \delta_{n, m}=\delta_{n, m} \quad \text { QED }
$$

Utilizing the notation for confluent hypergeometric function

$$
{ }_{0} F_{1}(-, 1+\alpha, x)=\sum_{n} \frac{1}{(1+\alpha)_{n}} \frac{x^{n}}{n !}
$$

we get from the previous formula the representation of Laguerre polynomials by an operator independent on the parameter

$$
x^{n} L_{n}\left(\frac{1}{x}\right)=\sum_{k=0}^{n}(-)^{k}\left(\begin{array}{l}
n \\
k
\end{array}\right) \frac{x^{n-k}}{k !}={ }_{0} F_{1}\left(-, 1,-D_{x}\right) x^{n}
$$

Under this form derivatives, recurrence relations, generating function for Laguerre polynomials are very simple to obtain.

For the generating function we have 


$$
\begin{aligned}
\circ \sum_{n=0}^{\infty} x^{n} L_{n}\left(\frac{1}{x}\right) t^{n}={ }_{0} F_{1}\left(-, 1,-D_{x}\right) \sum_{n=0}^{\infty} x^{n} t^{n} & ={ }_{0} F_{1}\left(-, 1,-D_{x}\right)(1-x t)^{-1} \\
& =(1-x t)^{-1} e^{-\frac{t}{1-x t}}
\end{aligned}
$$

Putting $x t=u, x=1 / z$ we get

$$
\text { ○ } \sum_{n=0}^{\infty} L_{n}(z) u^{n}==(1-u)^{-1} e^{-\frac{u z}{1-u}}
$$

(ii) The Generalized Laguerre Polynomials

Applying the operator $\left(1-D_{r}\right)^{-\alpha}$ onto the Laguerre differential equation and remarking by the fundamental identity that

$$
\left(1-D_{x}\right)^{-\alpha} \hat{X} \equiv \hat{X}\left(1-D_{x}\right)^{-\alpha}-\alpha\left(1-D_{x}\right)^{-\alpha-1}
$$

we get the differential equation of generalized Laguerre polynomials $L_{n}^{\alpha}(x)$

$$
\left(\hat{X} D_{x}^{2}+(\alpha+1-\hat{X}) D_{x}+n\right)\left(1-D_{x}\right)^{-\alpha} y=0
$$

and consequently

$$
L_{n}^{\alpha}(x)=\left(1-D_{x}\right)^{\alpha} L_{n}(x)=(-)^{n}\left(1-D_{x}\right)^{n+\alpha} \frac{x^{n}}{n !}
$$

With the notation $(a)_{n}=a(a+1) \ldots .(a+n-1)$ we have the explicit formulae

$$
\begin{aligned}
& L_{n}^{\alpha}(x)=\sum_{k=0}^{n}(-)^{k} \frac{(1+\alpha)_{n}}{(1+\alpha)_{k}} \frac{x^{k}}{(n-k) ! k !} \\
& L_{n}^{\alpha}(0)=(1+\alpha)_{n} / n !
\end{aligned}
$$

In particular

$$
\begin{aligned}
L_{n}^{-\frac{1}{2}}\left(x^{2}\right) & =(-)^{n} \sum_{k=0}^{n}(-)^{k} \frac{2^{-2 n}(2 n) !}{n !} \frac{(n-k) !}{2^{2 k-2 n}(2 n-2 k) !} \frac{x^{2 n-2 k}}{(n-k) ! k !} \\
= & \frac{(-)^{n}}{n !} \sum_{k=0}^{n}(-)^{k} \frac{1}{k ! 2^{2 k}} \frac{(2 n) !}{(2 n-2 k) !} x^{2 n-2 k}=\frac{(-)^{n}}{n !} \sum_{k=0}^{n} \frac{(-)^{k}}{k !} \frac{1}{2^{2 k}} D_{x}^{2 k} x^{2 n} \\
= & \frac{(-)^{n}}{n !} e^{-\frac{D_{x}^{2}}{4}} x^{2 n}=\frac{(-)^{n}}{n ! 2^{2 n}} H_{2 n}(x)
\end{aligned}
$$

By similar calculation we get also

$$
\text { ○ } x L_{n}^{1 / 2}\left(x^{2}\right)=\frac{(-)^{n}}{n ! 2^{2 n+1}} H_{2 n+1}(x)
$$

Utilizing the notation for confluent hypergeometric function we get the formula

$$
x^{n} L_{n}^{\alpha}\left(\frac{1}{x}\right)=(1+\alpha)_{n} F_{1}\left(-, 1+\alpha,-D_{x}\right) \frac{x^{n}}{n !}
$$

representing $x^{n} L_{n}^{\alpha}(1 / x)$ by a differential operator independent with respect to $\mathrm{n}$. From (4.65) and thank to the identity deduced from the fundamental one (3.15)

$$
\hat{X}^{m}\left(1-D_{x}\right)^{n+\alpha} \equiv \sum_{k=0}^{m} \frac{(1+\alpha)_{n}}{(1+\alpha)_{n-k}}\left(1-D_{x}\right)^{n+\alpha-k} \hat{X}^{m(k)}
$$

we get a recurrence relation between $x^{m} L_{n}^{\alpha}(x)$ and the $L_{n+m-k}{ }^{\alpha-m}(x)$, for example

$$
\circ \quad x L_{n}^{\alpha}(x)=-(n+1) L_{n+1}^{\alpha-1}(x)+(n+\alpha) L_{n}^{\alpha-1}(x)
$$


As for derivatives we have

$$
\text { ○ } \quad D_{x}^{m} L_{n}^{\alpha}(x)=(-)^{n}\left(1-D_{x}\right)^{n+\alpha} \frac{x^{n-m}}{(n-m) !}=(-)^{m} L_{n-m}{ }^{\alpha+m}(x)
$$

With the same remarks as for Hermite polynomials we get the addition formula

$$
\begin{aligned}
\circ \quad L_{n}^{\alpha}(x+y) & =(-)^{n}\left(1-D_{x}\right)^{n+\alpha} \frac{(x+y)^{n}}{n !}=(-)^{n}\left(1-D_{x}\right)^{a}\left(1-D_{y}\right)^{n+b} \frac{(x+y)^{n}}{n !} \\
& =\sum_{i=0}^{n} L_{i}^{a-i}(x) L_{n-i}^{b+i}(y) \quad a+b=\alpha
\end{aligned}
$$

The generating functions are obtained consicely by (4.66)

$$
\begin{aligned}
& \circ \sum_{n=0}^{\infty} \frac{1}{(1+\alpha)_{n}} x^{n} L_{n}{ }^{\alpha}\left(\frac{1}{x}\right) t^{n}={ }_{0} F_{1}\left(-, 1+\alpha,-D_{x}\right) \sum_{n=0}^{\infty} \frac{x^{n} t^{n}}{n !}={ }_{0} F_{1}(-, 1+\alpha,-t) e^{x t} \\
& \circ \quad \sum_{n=0}^{\infty} x^{n} L_{n}^{\alpha}\left(\frac{1}{x}\right) t^{n}={ }_{0} F_{1}\left(-, 1+\alpha,-D_{x}\right) \sum_{n=0}^{\infty}(1+\alpha)_{n} \frac{x^{n} t^{n}}{n !} \\
& \circ \sum_{n=0}^{\infty} \frac{n !}{(1+\alpha)_{n}} x^{n} L_{n}^{\alpha}\left(\frac{1}{x}\right) t^{n}={ }_{0} F_{1}\left(-, 1+\alpha,-D_{x}\right)(1-x t)^{-1} \\
&=F_{1}\left(1,1+\alpha,-\frac{t}{1-x t}\right)(1-x t)^{-1}
\end{aligned}
$$

Putting $x t=u, x=1 / z$ we get generating functions of $L_{n}^{\alpha}(x)$

$$
\begin{array}{ll}
\circ & \sum_{n=0}^{\infty} \frac{1}{(1+\alpha)_{n}} L_{n}^{\alpha}(z) u^{n}={ }_{1} F_{1}(-, 1+\alpha,-u z) e^{u t} \\
\circ & \sum_{n=0}^{\infty} L_{n}^{\alpha}(z) u^{n}=(1-u)^{-1-\alpha} e^{-\frac{u z}{1-u}} \\
\circ & \sum_{n=0}^{\infty} \frac{n !}{(1+\alpha)_{n}} L_{n}^{\alpha}(z) u^{n}={ }_{1} F_{1}\left(1,1+\alpha,-\frac{u z}{1-u}\right)(1-u)^{-1}
\end{array}
$$

(iv)The Gegenbauer polynomials

Defining the Gegenbauer polynomials by

$$
C_{n}^{\lambda}(x)=\sum_{m=0}^{\left[\frac{n}{2}\right]}(-)^{m} \frac{1}{m !(n-2 m) !}(\lambda)_{n-m}(2 x)^{n-2 m}
$$

and remarking that the operator $\hat{X}\left(\hat{X} D_{x}-n\right)$ vanishes $x^{n}$ and transforms $x^{n-2}$ into $(-2) x^{n-1}$ we may prove that

$$
\left(n x-x^{2} D_{x}\right) C_{n}^{\lambda}(x)=\sum_{m=0}^{\left[\frac{n}{2}\right]^{-1}}(-)^{m+1} \frac{1}{m !(n-2 m-2) !}(\lambda)_{n-m-1}(2 x)^{n-2 m-1}
$$

Besides we have $\quad D_{x} C_{n}^{\lambda}(x)=\sum_{0}^{\left[\frac{n}{2}\right]}(-)^{m} \frac{2(n-2 m)}{m !(n-2 m) !}(\lambda)_{n-m}(2 x)^{n-2 m-1}$

Combining these two formulae we get the recurrence relation

$$
\left(\left(1-x^{2}\right) D_{x}+n x\right) C_{n}^{\lambda}(x)=(2 \lambda-1+n) C_{n-1}^{\lambda}(x)
$$

By the fundamental identity (3.15) we may factorize

$$
\left(\left(1-\hat{X}^{2}\right) D_{x}+n \hat{X}\right) \equiv\left(1-\hat{X}^{2}\right)^{\frac{n}{2}+1} D_{x}\left(1-\hat{X}^{2}\right)^{-\frac{n}{2}}
$$

and get 


$$
\left(1-x^{2}\right)^{\frac{n}{2}+1} D_{x}\left(1-x^{2}\right)^{-\frac{n}{2}} C_{n}^{\lambda}(x)=(2 \lambda-1+n) C_{n-1}^{\lambda}(x)
$$

By iteration we obtain the differential equation of the Gegenbauer polynomials

$$
\left(\left(1-x^{2}\right)^{\frac{3}{2}} D_{x}\right)^{n}\left(1-x^{2}\right)^{-\frac{n}{2}} y_{n}=(2 \lambda)_{n} y_{0}
$$

Performing the change of argument

$$
\begin{aligned}
u(x)= & x\left(1-x^{2}\right)^{-\frac{1}{2}} \Rightarrow D_{u}=\left(1-x^{2}\right)^{\frac{3}{2}} D_{x} \\
& \left(1+u^{2}\right)=\left(1-x^{2}\right)^{-1} ; x^{2}=u^{2}\left(1+u^{2}\right)^{-1}
\end{aligned}
$$

we may change the equation (4.69) into

$$
D_{u}{ }^{n}\left(1+u^{2}\right)^{\frac{n}{2}} y_{n}=(2 \lambda)_{n} y_{0}
$$

and get the solution

$$
y_{n}=(2 \lambda)_{n}\left(1-x^{2}\right)^{\frac{n}{2}}\left(\left(1-x^{2}\right)^{\frac{3}{2}} D_{x}\right)^{-n} y_{0}
$$

i.e.

$$
y_{n}=(2 \lambda)_{n}\left(1+u^{2}\right)^{-\frac{n}{2}} D_{u}^{-n} y_{0}=(2 \lambda)_{n} y_{0}\left(1+u^{2}\right)^{-\frac{n}{2}} F\left(D_{u}\right) \frac{u^{n}}{n !}
$$

where $\mathrm{F}(x)$ is an arbitrary function $F(x)=\sum_{k=0}^{\infty} F_{k} x^{k}, \quad F_{0} \neq 0$.

Utilizing the fundamental identity (3.15) we have

$$
\left(1+u^{2}\right)^{-\frac{n}{2}} D_{u}\left(1+u^{2}\right)^{\frac{n}{2}}=D_{u}+\left(1+u^{2}\right)^{-1} \frac{n}{2} 2 u=D_{u}+\frac{n u}{\left(1+u^{2}\right)}
$$

so that the above equation may be written

$$
y_{n}=(2 \lambda)_{n} y_{0} F\left(D_{u}+\frac{n u}{1+u^{2}}\right)\left(1+u^{2}\right)^{-\frac{n}{2}} \frac{u^{n}}{n !}=(2 \lambda)_{n} y_{0} F\left(D_{u}+\frac{n u}{1+u^{2}}\right) \frac{x^{n}}{n !}
$$

Now from (4.73) and (4.70) we see that

$$
\begin{aligned}
\left(D_{u}+\frac{n u}{1+u^{2}}\right)^{k} & \frac{x^{n}}{n !} \\
= & \left(1+u^{2}\right)^{-\frac{n}{2}} D_{u}^{k}\left(1-x^{2}\right)^{-\frac{n}{2}} \frac{x^{n}}{n !}=\left(1+u^{2}\right)^{-\frac{n}{2}} D_{u}{ }^{k} \frac{u^{n}}{n !} \\
& \left(1+u^{2}\right)^{-\frac{n}{2}} \frac{u^{n-k}}{(n-k) !}=\left(1-x^{2}\right)^{\frac{n}{2}} \frac{x^{n-k}}{(n-k) !}\left(1-x^{2}\right)^{\frac{-n+k}{2}} \\
& =\left(1-x^{2}\right)^{\frac{k}{2}} \frac{x^{n-k}}{(n-k) !}=\left(1-x^{2}\right)^{\frac{k}{2}} D_{x}{ }^{k} \frac{x^{n}}{n !}
\end{aligned}
$$

This is a remarkable formula where the acting operator in the left hand side depends on the parameter $n$ although the one in the right doesn't.

Naming the operator in the right hand side by $B_{k}$

$$
B_{k} \equiv\left(1-x^{2}\right)^{\frac{k}{2}} D_{x}^{k}
$$

we get

$$
y_{n}=(2 \lambda)_{n} y_{0} \sum_{k=0}^{n} F_{k}\left(1-x^{2}\right)^{\frac{k}{2}} D_{x}{ }^{k} \frac{x^{n}}{n !}=(2 \lambda)_{n} y_{0} \sum_{k=0}^{n} F_{k} B_{k} \frac{x^{n}}{n !}
$$

and 


$$
y_{n}(0)=(2 \lambda)_{n} y_{0} F_{n}
$$

The Gegenbauer polynomials verify the initial condition

$$
y_{0}=1, \quad C_{2 n+1}^{\lambda}(0)=0, \quad C_{2 n}^{\lambda}(0)=(-)^{n} \frac{(\lambda)_{n}}{n !}
$$

so that

$$
F_{2 n}=\frac{C_{2 n}^{\lambda}(0)}{(2 \lambda)_{2 n}}=(-)^{n} \frac{(\lambda)_{n}}{n !(2 \lambda)_{2 n}} \quad F_{2 n+1}=0
$$

i.e.

$$
\begin{array}{r}
F(x)={ }_{1} F_{1}\left(\lambda ; 2 \lambda ;-x^{2}\right)={ }_{0} F_{1}\left(-; \lambda+\frac{1}{2} ;-\frac{x^{2}}{4}\right) \\
C_{n}^{\lambda}(x)=(2 \lambda)_{n} \sum_{k=0}^{\left[\frac{n}{2}\right]}(-)^{k} \frac{(\lambda)_{k}}{k !(2 \lambda)_{2 k}}\left(1-x^{2}\right)^{k} D_{x}^{2 k} \frac{x^{n}}{n !}
\end{array}
$$

Finally we obtain the symbolic representation of Gegenbauer polynomials

$$
C_{n}^{\lambda}(x)=:(2 \lambda)_{n}{ }_{0} F_{1}\left(-; \frac{1}{\lambda+1 / 2} ;-\frac{B^{2}}{4}\right) \frac{x^{n}}{n !}
$$

where the undefined notation $B^{2 k}$ must be replaced with the well defined operator $B_{2 k}$.

The representation of Gegenbauer polynomials by a differential operator is easy to remember, convenient for searching differential recurrence relations.

The generating function may also be calculated as followed where we put for simplicity $F(B)={ }_{1} F_{1}\left(\lambda ; 2 \lambda ;-\frac{B^{2}}{4}\right)$

$$
\begin{aligned}
\sum_{n=0}^{\infty} C_{n}^{\lambda}(x) t^{n} & =: F(B) \sum_{n=0}^{\infty}(2 \lambda)_{n} \frac{(x t)^{n}}{n !}=: F(B)(1-x t)^{-2 \lambda} \\
& =\sum_{k=0}^{\infty} F_{2 k} B_{2 k}(1-x t)^{-2 \lambda}=\sum_{k=0}^{\infty} F_{2 k}\left(1-x^{2}\right)^{k} D^{2 k}(1-x t)^{-2 \lambda} \\
& =\sum_{k=0}^{\infty} F_{2 k}\left(1-x^{2}\right)^{k}(2 \lambda)_{2 k} t^{2 k}(1-x t)^{-2 \lambda-2 k} \\
& =(1-x t)^{-2 \lambda} \sum_{k=0}^{\infty}(-)^{k} \frac{(\lambda)_{k}}{k !} \frac{\left(1-x^{2}\right)^{k} t^{2 k}}{(1-x t)^{2 k}} \\
& =(1-x t)^{-2 \lambda}\left(1+\frac{\left(1-x^{2}\right) t^{2}}{(1-x t)^{2}}\right)^{-\lambda}=\left(1+2 x t+t^{2}\right)^{-\lambda}
\end{aligned}
$$

The Legendre polynomial $P_{n}(x)$ is a particular case of Gegenbauer polynomials corresponding to $\lambda=1 / 2$ so that

$$
P_{n}(x)=:_{0} F_{1}\left(-; 1 ;-\frac{B^{2}}{4}\right) x^{n}=\sum_{k=0}^{n}(-)^{k} \frac{B_{2 k}}{4 k ! k !} x^{n}=\sum_{k=0}^{n}\left(x^{2}-1\right)^{k} \frac{D_{x}^{2 k}}{4 k ! k !} x^{n}
$$

(v) The Chebyshev polynomials

The Chebyshev polynomials of the first kind $T_{n}(x)$ are related to the Gegenbauer polynomials by the relation

$$
T_{n}(x)=\lim _{\lambda \rightarrow 0} \frac{n}{2 \lambda} C_{n}{ }^{\lambda}(x)=: n !_{0} F_{1}\left(-; \frac{1}{1 / 2} ;-\frac{B^{2}}{4}\right) \frac{x^{n}}{n !}
$$

so that we get a very simple representation of them

$$
T_{n}(x)=: \cos B x^{n}
$$

From this formula we may deduce some generating functions of $T_{n}(x)$ 


$$
\begin{aligned}
\circ \quad \sum_{n=0}^{\infty} T_{n}(x) \frac{t^{n}}{n !} & =: \cos B e^{t x}=\sum_{k=0}^{\infty}(-)^{k} \frac{1}{(2 k) !}\left(1-x^{2}\right)^{k} t^{2 k} e^{t x}=\cosh \mathrm{t} \sqrt{\mathrm{x}^{2}-1} e^{t x} \\
\circ \quad \sum_{n=0}^{\infty} T_{n}(x) t^{n} & =: \cos B \frac{1}{1-x t}=\sum_{k=0}^{\infty} \frac{1}{(2 k) !}\left(x^{2}-1\right)^{k} D_{x}^{2 k} \frac{1}{1-x t}=\frac{1-x t}{1-2 x t+t^{2}} \\
\circ \quad \sum_{n=1}^{\infty} T_{n}(x) \frac{t^{n}}{n} & =: \cos B\left(\ln \frac{1}{1-x t}-1\right)=\sum_{k=0}^{\infty} \frac{1}{(2 k) !}\left(x^{2}-1\right)^{k} D_{x}^{2 k} \ln \frac{1}{1-x t} \\
& =\ln \frac{1}{1-x t}+\sum_{k=1}^{\infty}\left(x^{2}-1\right)^{k} t^{2 k}(1-x t)^{-2 k}=\ln \frac{1}{\sqrt{1-2 x t+t^{2}}}
\end{aligned}
$$

and the important properties coming trom (4.82)

- $T_{n}(x)=\sum_{k=0}^{\left[\frac{n}{2}\right]} \frac{1}{(2 k) !}\left(x^{2}-1\right)^{k} D_{x}^{2 k} x^{n}=\sum_{k=0}^{\left[\frac{n}{2}\right]}\left(\begin{array}{c}n \\ 2 k\end{array}\right)\left(x^{2}-1\right)^{k} x^{n-2 k}$

- $\quad T_{n}(\cos \theta)=\sum_{k=0}^{\left[\frac{n}{2}\right]}\left(\begin{array}{c}n \\ 2 k\end{array}\right)(i \sin \theta)^{2 k} \cos ^{n-2 k} \theta=\operatorname{Re}(\cos \theta+\operatorname{isin} \theta)^{n}=\cos n \theta$

- $T_{n}\left(T_{m}(\cos \theta)\right)=T_{n}(\cos m \theta)=\sum_{k=0}^{\left[\frac{n}{2}\right]}\left(\begin{array}{c}n \\ 2 k\end{array}\right)(i \sin m \theta)^{2 k} \cos ^{n-2 k} m \theta$

$$
=\cos n m \theta=T_{n m}(\cos \theta)
$$

○ $T_{n}\left(T_{m}(x)\right)=T_{n m}(x)$

Similarly the Chebyshev polynomials of the second kind $U_{n}(x)$ are defined by

$$
U_{n}(x)=C_{n}^{1}(x)=:(n+1) \frac{\sin B}{B} x^{n}
$$

From this formula we get

$$
\begin{gathered}
U_{n}(x)=(n+1) \sum_{k=0}^{\left[\frac{n}{2}\right]} \frac{n !}{(2 k+1) !(n-2 k) !}\left(x^{2}-1\right)^{k} x^{n-2 k} \\
\circ \quad U_{n}(\cos \theta)=(\sin \theta)^{-1} \sum_{k=0}^{\left[\frac{n}{2}\right]} \frac{(-)^{k}(n+1) !}{(2 k+1) !(n+1-2 k-1) !}(i \sin \theta)^{2 k+1} \cos ^{n+1-2 k-1} \theta \\
=\sin ^{-1} \theta \operatorname{Im}(\operatorname{isin} \theta+\cos \theta)^{\mathrm{n}+1}=\sin ^{-1} \theta \sin (n+1) \theta
\end{gathered}
$$

and some generating functions.

Searching for relations between $T_{n}(x)$ and $U_{n}(x)$ we utilize the fundamental identity (3.15) to get

$$
\begin{aligned}
& D_{x}\left(1-\hat{X}^{2}\right)^{k} D_{x}^{2 k} \equiv\left(\left(1-\hat{X}^{2}\right)^{k} D_{x}-2 k \hat{X}\left(1-\hat{X}^{2}\right)^{k-1}\right) D_{x}^{2 k} \\
& {\left[D_{x}, B_{2 k}\right] \equiv-2 k \hat{X} B_{2 k-2} D_{x}^{2}} \\
& {\left[\hat{X}, B_{2 k}\right] \equiv-2 k B_{2 k-2} D_{x}} \\
& \left.\left[\hat{X} D_{x}, B_{2 k}\right] \equiv\left(-2 k \hat{X}^{2}-2 k\right) B_{2 k-2} D_{x}\right) D_{x}
\end{aligned}
$$

and find 


$$
\begin{array}{ll}
\circ & \sin \mathrm{B} \equiv:(1-\hat{X})^{\frac{1}{2}} \frac{\sin B}{B} D_{x} \\
\circ & {\left[D_{x}, \cos \mathrm{B}\right] \equiv: \hat{X} \frac{\sin B}{B} D_{x}{ }^{2} \equiv: \hat{X}\left(1-\hat{X}^{2}\right)^{-\frac{1}{2}} \sin B D_{x}} \\
\circ \quad & D_{x} \cos \mathrm{B} \equiv: \frac{\sin B}{B} D_{x} \hat{X} D_{x} \equiv:\left(1-\hat{X}^{2}\right)^{-\frac{1}{2}} \sin B \hat{X} D_{x} \\
\circ \quad[\hat{X}, \cos \mathrm{B}] \equiv:\left(1-\hat{X}^{2}\right) \frac{\sin B}{B} D_{x} \equiv:\left(1-\hat{X}^{2}\right)^{\frac{1}{2}} \sin B \\
\circ \quad[\hat{X}, \sin \mathrm{B}] \equiv:-\left(1-\hat{X}^{2}\right)^{\frac{1}{2}} \cos B
\end{array}
$$

From these identities we obtain

$$
\begin{array}{ll}
\circ & U_{n}(x)=:(n+1) \frac{\sin B}{B} x^{n}=\frac{\sin B}{B} D_{x} x^{n+1}=\left(1-x^{2}\right)^{-\frac{1}{2}} \sin B x^{n+1} \\
\circ & D_{x} T_{n}(x)-n T_{n-1}(x)=n x U_{n-2}(x) \\
\circ & D_{x} T_{n}(x) \equiv: \frac{\sin B}{B} D_{x} \hat{X} D_{x} \equiv:\left(1-\hat{X}^{2}\right)^{-\frac{1}{2}} \sin B \hat{X} D_{x}=n U_{n-1}(x) \\
\circ & x T_{n}(x)-T_{n+1}(x)=\left(1-x^{2}\right) U_{n-1}(x) \\
\circ & x U_{n-1}(x)-U_{n}(x)=-T_{n}(x)
\end{array}
$$

4.7 The Laplace Transform

From the definition of the Laplace transform

$$
F(x)=\int_{0-}^{\infty} e^{-x t} f(t) d t
$$

we may write at least for entire functions defined in the interval $\left[0^{-}+\infty\right]$

$$
F(x)=\int_{0-}^{\infty} f\left(-D_{x}\right) e^{-x t} d t=f\left(-D_{x}\right) \frac{1}{x}
$$

From the above differential representation of the Laplace transform we get

$$
\begin{aligned}
& \circ f(x)=x^{n} \Rightarrow F(x)=\left(-D_{x}\right)^{n} \frac{1}{x}=n ! x^{-n-1} \\
& \circ f(x)=e^{a x} \Rightarrow F(x)=e^{-a D_{x}} \frac{1}{x}=\frac{1}{x-a} \\
& \circ f(x)=e^{i \omega x} \Rightarrow F(x)=\frac{1}{x-i \omega}=\frac{x+i \omega}{x^{2}+\omega^{2}}
\end{aligned}
$$

The tranforms of $\cos \omega x$ and $\sin \omega x$ are deduced from the above formula.

Utilizing the notation $L f(x)$ to denote the Laplace transform of $f(x)$ we get

$$
\begin{aligned}
& \circ \quad L f(a x)=f\left(-a D_{x}\right) \frac{1}{x}=f\left(-D_{u}\right) \frac{1}{a u}=\frac{1}{a} L f(u)=\frac{1}{a} L f\left(\frac{x}{a}\right) \quad x=a u, a>0 \\
& \circ \quad L x^{n} f(x)=\left(-D_{x}\right)^{n} f\left(-D_{x}\right) \frac{1}{x}=\left(-D_{x}\right)^{n} L f(x) \\
& \circ \quad L x^{-1} f(x)=\left(-D_{x}\right)^{-1} f\left(-D_{x}\right) \frac{1}{x}=\left(-D_{x}\right)^{-1} L f(x)+\left(-D_{x}\right)^{-1} 0=\int_{x}^{\infty} L f(t) d t
\end{aligned}
$$

The integral limits are choosen so that the function in the left member converges at infinity. 


$$
\text { ○ } L f(x-a)=f\left(-D_{x}-a\right) \frac{1}{x}=e^{-a x} f\left(-D_{x}\right) e^{a x} \frac{1}{x}
$$

Now, by the fundamental identity we have

$$
\begin{aligned}
& \hat{X} f\left(-D_{x}\right) \equiv f\left(-D_{x}\right) \hat{X}+f^{(1)}\left(-D_{x}\right) \\
& \hat{X} f^{(1)}\left(-D_{x}\right) \equiv f^{(1)}\left(-D_{x}\right) \hat{X}+f^{(2)}\left(-D_{x}\right) \\
& (\ldots) \\
& \hat{X} f^{(n-1)}\left(-D_{x}\right) \equiv f^{(n-1)}\left(-D_{x}\right) \hat{X}+f^{(n)}\left(-D_{x}\right)
\end{aligned}
$$

Combining these $\mathrm{n}$ formulae we get

$$
\hat{X}^{n} f\left(-D_{x}\right) \equiv \sum_{k=0}^{n-1} \hat{X}^{k} f^{(n-k-1)}\left(-D_{x}\right) \hat{X}+f^{(n)}\left(-D_{x}\right)
$$

Applying this identity onto the function $\frac{1}{x}$ we obtain the famous formula

$$
\text { ○ } L f^{(n)}(x)=x^{n} L f(x)-\sum_{k=0}^{n-1} x^{k} f^{(n-k-1)}(0)
$$

Replace $f(x), f^{\prime}(x)$ with $\int f(x), f(x)$ and take $\mathrm{n}=1$ we get the Laguerre transform of $\int_{0}^{x} f(t) d t$

$$
\begin{aligned}
& L f(x)=x L \int f(x)-\left.\int f(x)\right|_{x=0} \\
& \circ \quad \frac{1}{x} L f(x)=L \int_{0}^{x} f(t) d t
\end{aligned}
$$

Let $H(x)$ be the Heaviside function, $H(x)=0$ for $x<0 ; H(x)=1$ for $x>0$ we have by factorizing $\left(D_{x}+a \hat{I}\right)$ into

$$
\begin{aligned}
& e^{-a \hat{X}} D_{x} e^{a \hat{X}} \\
& \circ \quad L f(x-a) H(x-a)=\int_{a}^{\infty} e^{-x t} f(t-a) d t=f\left(-D_{x}-a\right) \int_{a}^{\infty} e^{-x t} d t=f\left(-D_{x}-a\right) \frac{e^{-a x}}{x} \\
& \circ \quad=e^{-a x} f\left(-D_{x}\right) e^{a x} \frac{e^{-a x}}{x}=e^{-a x} L f(x)
\end{aligned}
$$

From the above formula we get

$$
\begin{gathered}
\quad L \int_{0}^{\infty} f\left(x_{0}\right) g\left(x-x_{0}\right) H\left(x-x_{0}\right) d x_{0}=\int_{0}^{\infty} f\left(x_{0}\right) e^{-x x_{0}} \operatorname{Lg}(x) d x_{0} \\
=(\operatorname{Lf}(x))(\operatorname{Lg}(x)) \\
\circ \quad L \int_{0}^{x} f\left(x_{0}\right) g\left(x-x_{0}\right) d x_{0}=(\operatorname{Lf}(x))(\operatorname{Lg}(x))
\end{gathered}
$$

\section{Remarks and Conclusions}

Theoretically speaking, this work contributes to the development of differential calculus by a definition of the inverse operator of an arbitrary operator, including the ones having nonvanishing kernel.

The second contribution of this work consists in proposing an identity, qualified fundamental, which changes the operators $f(A) g(B)$ where $A B-B A \equiv \hat{I}$ into a sum of operators $\frac{1}{k !} g^{(k)}(B) f^{(k)}(A)$. Practical applications of such identities in many situations lead to a new approach for resolution of differential equations; for representing many special functions as the transforms of monomials by differential operators and obtaining afterward in a 
very concise manner all main properties of these polynomials. At the end we propose an elegant differential form for the Laplace transform and prove very concisely thank to the fundamental identity most of its properties.

The last but not least conclusion is that the matter in this work is relatively readable, self - consistent so that it is ready for teaching to students.

\section{Acknowledgments}

The author dedicates with love and respect this work to his father Do Huu Quon for the education and high attention he had given him and to his beautiful Fatherland Vietnam.

He is gratefully toward the Université libre de Bruxelles and the Université de l'Etat à Mons for octroying him many and many opportunities to develop his task of resolving mathematical problems met in theoretical physics.

\section{Reference}

Heaviside, O. (1893). On Operators in Physical Mathematics Part II. Proc. Roy. Soc., 54, 105-143.

Forsyth, A. R. (1888). A treatise of differential equations. London: McMilan and Cie and New York.

Eckart, C. (1926). Operator calculus and the solutions of the equations of quantum dynamics. Phys. Rev., 28, 711-26. http://dx.doi.org/10.1103/PhysRev.28.711

Abramovitz, M., \& Stegun, I. A. (1968). Handbook of Mathematical Functions. New York: Dover.

Wilcox, R. M. (1967). Exponential operators and parameters differentiation in quantum physics. J. Mathematical Phys., 8, 962-982. http://dx.doi.org/10.1063/1.1705306

Do, T. S. (1978). Representation of Special functions by differintegral and hyperdifferential operators. SIAM $J$ Math Anal, 9(6), 1068-1075

Oldham, K. B., \& Spanier, J. (1974). The fractional calculus. New York, London: Academic Press.

Wolf, K. B. (1979). Integral transforms in Science and Engineering. New York: Plenum Press. http://dx.doi.org/10.1007/978-1-4757-0872-1

Do, T. S. (2015). Differential transforms. The case of the Fourier transform. J. Applied Physics Research, 7(2), 137-153. http://dx.doi.org/10.5539/apr.v7n2p137

\section{Copyrights}

Copyright for this article is retained by the author(s), with first publication rights granted to the journal.

This is an open-access article distributed under the terms and conditions of the Creative Commons Attribution license (http://creativecommons.org/licenses/by/3.0/). 